\title{
Protein Kinase D promotes activity-dependent AMPA receptor endocytosis in hippocampal neurons
}

\author{
1 Carlos O. Oueslati Morales ${ }^{1}$, Attila Ignácz ${ }^{2}$, Norbert Bencsik ${ }^{2}$, Anikó Erika Rátkai ${ }^{2}$, Wolfgang \\ 2 S. Lieb ${ }^{1}$, Stephan A. Eisler ${ }^{1,3}$, Attila Szücs ${ }^{2}$, Katalin Schlett ${ }^{2}$, Angelika Hausser ${ }^{1,3^{*}}$ \\ $3 \quad{ }^{1}$ Membrane Trafficking and Signalling Group, Institute of Cell Biology and Immunology, University \\ 4 of Stuttgart, Stuttgart, Germany \\ $5 \quad{ }^{2}$ Neural Cell Biology Group, Department of Physiology and Neurobiology, Eötvös Loránd \\ 6 University, Budapest, Hungary \\ $7 \quad{ }^{3}$ Stuttgart Research Center Systems Biology, University of Stuttgart, Stuttgart, Germany \\ 8 *Correspondence: \\ 9 Angelika Hausser \\ 10 angelika.hausser@izi.uni-stuttgart.de
}

11 Keywords: AMPA receptor, GluA1, endocytosis, chemically induced LTD, Rabaptin5, Rab5

12 Abstract

13 AMPA type glutamate receptors (AMPARs) mediate the majority of fast excitatory neurotransmission 14 in the brain. The continuous trafficking of AMPARs into and out of synapses is a core feature of synaptic plasticity, which is considered as the cellular basis of learning and memory. The molecular mechanisms underlying the postsynaptic AMPAR trafficking, however, are still not fully understood. In this work, we demonstrate that the Protein Kinase D (PKD) family promotes basal and activityinduced AMPAR endocytosis in primary hippocampal neurons. Pharmacological inhibition of PKD increased synaptic levels of GluA1-containing AMPARs, slowed down their endocytic trafficking and increased neuronal network activity. By contrast, ectopic expression of constitutive active PKD decreased the synaptic level of AMPARs, while increasing their co-localization with early endosomes. On a molecular level, we identify phosphorylation of the Rab5 effector protein and PKD substrate Rabaptin-5 to be required for promoting activity-dependent AMPAR endocytosis. Our results thus establish an important role for PKD in the regulation of postsynaptic AMPAR trafficking during synaptic plasticity.

\section{Introduction}

Synaptic plasticity describes a process where synaptic strength is changed in an activity-dependent manner within the brain. The most widely investigated forms of long-term synaptic changes include long-term potentiation (LTP) and long-term depression (LTD), which constitute the cellular basis of learning and memory (Huganir and Nicoll 2013, Malinow and Malenka 2002). One of the key elements in the control of synaptic strength is the dynamic alteration in the number and composition of the ionotropic AMPA-type glutamate receptors (AMPARs) within the postsynaptic membrane (Chater and Goda 2014, Huganir and Nicoll 2013, Malinow and Malenka 2002). 
AMPARs are tetramers composed of four different subunits, GluA1-GluA4. Binding of glutamate allows the opening of the ion channel and the subsequent influx of $\mathrm{Na}^{+}$(and potentially $\mathrm{Ca}^{2+}$ ) and efflux of $\mathrm{K}^{+}$, causing membrane depolarization. Thus, the main function of AMPARs is to mediate excitatory neurotransmission in the brain (Chater and Goda 2014, Diering and Huganir 2018). The heterotetramer GluA1/2 is the most abundant variant followed by GluA2/3, while the GluA4 subunit is expressed mainly during early development and is present only at low levels in the adult brain (Henley and Wilkinson 2013, Lu et al. 2009, Zhu et al. 2000). GluA1-containing AMPARs leave the endoplasmic reticulum rapidly and are trafficked towards the synaptic plasma membrane. Plasma membrane insertion is thought to happen mostly within the extra- or perisynaptic regions, followed by a lateral movement of AMPARs into the synapse. Within the synaptic membrane, AMPARs are stabilized by postsynaptic density (PSD) scaffolding proteins in an activity-dependent manner (Greger et al. 2002, Henley and Wilkinson 2013, Makino and Malinow 2009, Pick and Ziff 2018). Mature AMPARs are constitutively recycled through endocytic trafficking pathways: AMPARs are internalized from the cell surface at so-called Endocytic Zones localized adjacent to the PSD. Subsequently, AMPARs are trafficked to early endosomes and either recycled back to the plasma membrane or transported to dendritic lysosomes and degraded (Blanpied et al. 2002, Ehlers 2000, Luscher et al. 1999, van der Sluijs and Hoogenraad 2011). During LTD and LTP, endocytotic and exocytotic AMPAR trafficking is imbalanced, resulting in reduced and enhanced numbers of AMPARs at the synapse, respectively (Carroll et al. 1999, Ehlers 2000, Hayashi et al. 2000, Shi et al. 1999).

The protein kinase D (PKD) family of serine/threonine kinases belongs to the calmodulin-dependent protein kinases (CaMKs) superfamily, and comprises three isoforms in mammals: PKD1, PKD2 and PKD3, all of which are expressed in neurons from a very early embryonic stage (Ellwanger and Hausser 2013, Oster et al. 2006). PKDs are recruited to the plasma membrane or to different organelles, such as the Golgi complex, by binding to diacylglycerol. Upon activation, they control different intracellular processes such as vesicle fission from the Golgi complex and rearrangement of the actin cytoskeleton (reviewed in (Olayioye et al. 2013, Reinhardt et al. 2020)). In breast cancer cells, PKD-mediated phosphorylation of Rabaptin-5, a binding partner for both Rab4 and Rab5, is required to shunt integrin $\alpha_{v} \beta_{3}$ to the short recycling loop, indicating a role for the kinase in endocytic trafficking processes (Christoforides et al. 2012, Woods et al. 2004). In neurons, PKD plays a key role in the establishment and maintenance of polarity through controlling the integrity of the Golgi complex and trans-Golgi network-derived sorting of vesicles (Bisbal et al. 2008, Czondor et al. 2009, Yin et al. 2008). Furthermore, we recently demonstrated a role for PKD in learning and memory through stabilization of plasticity-induced actin rearrangements (Bencsik et al. 2015). However, so far PKD has not been linked to AMPAR trafficking in neuronal cells.

Here, we identify PKD as a regulator of basal and activity-mediated GluA1 endocytosis in cultured hippocampal neurons. We provide evidence that PKD is activated downstream of chemically induced GluA1 endocytosis. Inhibition of PKD increases surface and synaptic levels of GluA1 and consequently, neuronal network activity. We further show that expression of constitutive active PKD (caPKD) decreases the synaptic amount of GluA1 while increasing the localization of GluA1 at early endosomes. Importantly, PKD inhibition blocks the endocytosis of GluA1 upon chemically evoked LTD (cLTD). On a molecular level, we identify the Rab5 effector protein and PKD substrate Rabaptin5 to be involved in endocytosis of GluA1. Combined, our data identify a previously unknown role of the PKD family in the regulation of AMPAR endocytosis in cultured hippocampal neurons. 


\section{Animal handling}

CD1 mice (Charles River) were kept in the animal facility of the Institute of Cell Biology and Immunology, University of Stuttgart; or of the Biological Institute, Eötvös Loránd University, at $22 \pm$ $1{ }^{\circ} \mathrm{C}$ with $12 \mathrm{~h}$ light and dark cycles and ad libitum access to water and food. The animals were maintained and handled in accordance with the Guidelines for Accommodation and Care of Animals, according to the European Convention for the Protection of Vertebrate Animals Used for Experimental and Other Scientific Purposes.

\section{Cell culture}

Primary cultures of embryonic hippocampal cells were obtained from CD1 mice on embryonic day E17-E18 according to a published protocol (Czondor et al. 2009). In brief, cells were plated on polyL-lysine-coated (PLL, Sigma \#5899) 6 well plates (Greiner Bio-One, \#657160) or $35 \mathrm{~mm}$ petri dishes (Greiner Bio-One, \#627160) at a density of $4 \times 10^{5}$ cells/well. For imaging, cells were seeded on PLL/laminin-coated (Sigma; \#L2020) glass coverslips (Carl Roth, \#YX03.1) in 24-well plates or on glass bottom petri-dishes (Greiner Bio-One, \#627870) at a density of $1.15 \times 10^{5}$ cells/well. Five days after plating, half of the original NeuroBasal culture medium (ThermoFisher Scientific, \#21103049) supplemented with B27 (ThermoFisher Scientific, \#17504044) was changed to Brainphys (Stemcell technologies, \#05790) supplemented with SM1 (Stemcell technologies, \#05711), gentamycin (Sigma, \#G1397) and amphotericin B (Gibco, \#15290-026), containing $10 \mu \mathrm{M}$ cytosin-arabinofuranoside (Sigma, \#C6645). The cells were cultivated for 13 days at $37^{\circ} \mathrm{C}$ in $5 \% \mathrm{CO}_{2}$, and one half of the Neurobasal media was exchanged by fresh supplemented Brainphys every 3 days. HEK293T cells were cultivated in RPMI medium (Gibco, \#21875-034) supplemented with 10\% fetal bovine serum (FBS, Pan Biotech, \#P30-3309). Chemically-induced Long Term Depression (cLTD) was induced by treating the neuronal cultures with $50 \mu \mathrm{M}$ NMDA (Sigma, \#M3262) for $5 \mathrm{~min}$ in conditioned medium. Then, cells were changed to a NMDA-free medium, and incubated for $15 \mathrm{~min}$ before being further processed. Agonist-induced AMPAR endocytosis was performed by treating the neuronal cultures with $100 \mu \mathrm{M}$ S-AMPA (Hello Bio, \#HB0052) and $50 \mu \mathrm{M}$ D-AP5 (Tocris \#3693) for 2 minutes in conditioned medium. Afterwards, cells were changed to an AMPA- and D-AP5-free medium and incubated for 10 minutes before being further processed.

\section{Chemical treatments, antibody feeding and cell surface biotinylation}

PKD activity was blocked with CRT0066101 (CRT, Tocris \#4975) (Harikumar et al. 2010) diluted in dimethyl sulfoxide (DMSO), at a final concentration of $2 \mu \mathrm{M}$. For surface biotinylation of endogenous GluA1, 13-day-old neuronal cultures were placed on ice (13 days in vitro; DIV13), washed twice with calcium- and magnesium-containing phosphate-buffered saline (PBSCM, ThermoFisher Scientific, \#14040-091) and incubated with $1.5 \mathrm{mg} / \mathrm{ml}$ sulfo-NHS-SS-biotin (ThermoFisher Scientific, \#21331) diluted in PBSCM for 15 min. Afterwards, cultures were washed twice with $20 \mathrm{mM}$ glycine (Roth, \#HN07.1) in PBSCM for $7 \mathrm{~min}$, to bind free biotin; before being finally lysed. To visualize surface GluA1, we employed an antibody feeding assay modified from a published protocol (Sziber et al. 2017). In brief, neuronal cultures were incubated with an N-terminal-specific GluA1 antibody (1:100, Merck, \#MAB2263) in conditioned media for $10 \mathrm{~min}$ at $37^{\circ} \mathrm{C}$ and $5 \% \mathrm{CO}_{2}$ in a humidified atmosphere. Afterwards, cells were washed with PBS and fixed with 4\% paraformaldehyde (PFA) in PBS for 10 min at room temperature (RT) without permeabilization. After $1 \mathrm{~h}$ of blocking with $5 \%$ FBS and $0.1 \%$ $\mathrm{NaN}_{3}$ in PBS, a secondary anti-mouse antibody labelled with Alexa 546 (1:500, Invitrogen, \#A11030) 
was applied to the cells for $1 \mathrm{~h}$ at RT. Cells were washed and fixed again with $4 \%$ PFA in PBS for 10 min and further processed for Shank2 staining (see section Immunostaining and confocal laser scanning microscopy). Samples were mounted in ProLong Gold Antifade Mountant (ThermoFisher Scientific, \#P36930) and analyzed by confocal laser scanning microscopy.

To analyze the amount of endocytosed AMPAR, biotinylation assays of receptor internalization were employed as described (Ehlers 2000). In brief, protein lysosomal degradation was inhibited with 100 $\mu \mathrm{g} / \mathrm{ml}$ Leupeptin (Tocris, \#1167) for $1 \mathrm{~h}$; then cells were placed on ice and incubated with $1.5 \mathrm{mg} / \mathrm{ml}$ Sulfo-NHS-SS-biotin (ThermoFisher Scientific, \#21331) for $1 \mathrm{~h}$. Subsequently, cells were transferred to biotin-free medium and treated with $100 \mu \mathrm{m} \mathrm{S-AMPA}$ and $50 \mu \mathrm{M}$ D-AP5 for $2 \mathrm{~min}$. Then, cells were returned to an AMPA- and D-AP5 free medium for $10 \mathrm{~min}$ to allow for endocytosis, subsequently placed again on ice and washed twice with cold PBSCM. Remaining surface biotin was cleaved by incubation with glutathione cleavage buffer (50 nM L-Glutathione (Sigma \#G6013, $75 \mathrm{mM} \mathrm{NaCl}, 10$ mM EDTA, 1\% BSA, pH 8.6) for 15 min. Finally, cells were washed twice and further processed for protein extraction.

\section{Protein extraction, biotin pulldowns and Western Blotting}

Neuronal cells were harvested in cold lysis buffer (1\% Triton X-100, $20 \mathrm{mM}$ Tris $\mathrm{pH} 7.5,150 \mathrm{mM}$ $\mathrm{NaCl}, 1 \mathrm{mM}$ EDTA, $1 \mathrm{mM}$ EGTA; $4^{\circ} \mathrm{C}$ ) supplemented with protease (Roche, \#116974988001) and phosphatase inhibitors (Roche, \#04906845001), and subsequently centrifugated at $13000 \mathrm{x}$ g for 10 min. For the biotin pulldowns, equal amounts of cell lysate were incubated with avidin-coated agarose beads (Thermo Scientific, \#29201) for $90 \mathrm{~min}$ at $4^{\circ} \mathrm{C}$ and washed 4 times with lysis buffer. HEK293T cells were harvested in homogenization buffer $(300 \mathrm{mM} \mathrm{NaCl}, 10 \mathrm{mM} \mathrm{MgCl} 2,10 \%$ glycerol, $20 \mathrm{mM}$ HEPES, $0.1 \%$ NP-40 and $1 \mathrm{mM}$ DTT) supplemented with protease and phosphatase inhibitors, and subsequently centrifuged at $9300 \mathrm{G}$ for $3 \mathrm{~min}$. To determine Rab5 activity, equal amounts of cell lysate were incubated with GST-tagged Rabaptin-5:Rab5 Binding Domain beads (Qi et al. 2015) for 60 min at $4^{\circ} \mathrm{C}$ and then washed once with homogenization buffer. Equal amounts of protein were run on NuPage Novex 4-12\% Bis-Tris gels (Thermo Fisher Scientific, \#NP0322BOX) and blotted onto nitrocellulose membranes using the iBlot device (Thermofisher scientific, \#IB1001). Membranes were blocked for $30 \mathrm{~min}$ with $0.5 \%$ (v/v) blocking reagent (Roche, \#11096176001) in PBS containing 0.05\% Tween 20 and $0.1 \% \mathrm{NaN}_{3}$, and subsequently incubated with primary antibodies overnight at $4{ }^{\circ} \mathrm{C}$ (antiPKD1 (1:1000, Cell Signaling Technologies, CST, \#2052), anti-pS916PKD (1:1000, CST, \#2051), anti-actin (1:1000, Sigma, \#A4700), anti-GluA1 (1:1000, Merck, \#MAB2263), anti-pS831GluA1 (1:1000, CST, \#75574), anti-GFP (1:2000, Roche, \#11814460001)). The next day, membranes were washed and incubated with HRP-conjugated secondary anti-mouse and anti-rabbit antibodies at RT for $1 \mathrm{~h}$ (1:10000, Dianova, \#115-035-062 or \#111-035-144) before being washed again and finally visualized using the SuperSignal West Pico PLUS or the SuperSignal West Dura Extended Duration substrates (Thermo Fisher Scientific, \#34580 or \#34076). Proteins were visualized using the Amersham Imager 600, an enhanced chemiluminescence detection system (Thermo Fisher Scientific, Waltham, MA, USA). Quantitative Western Blotting chemiluminescence was detected at a depth of 16-bit in the linear detection range of the device, equipped with a 3.2-megapixel super-honeycomb CCD camera fitted with a large aperture f/0.85 FUJINON lens. Special care was taken not to overexpose in order to guarantee accurate quantifications. Densitometry was performed using Image Studio Lite 5.2 (Li-COR Biosciences, Bad Homburg, Germany). For each protein, the integrated density of the signal was measured, corrected for background signals and adjusted to loading controls. 


\section{Transfection and expression constructs}

168

169

170

171

172

173

174

175

176

177

178

179

180

181

182

183

184

185

186

187

188

189

190

191

192

193

194

195

196

197

198

199

200

201

202

203

204

205

206

207

208

209

210

On DIV12, neuronal cultures were transfected using Lipofectamine 2000 (Invitrogen, \#11668-019) according to the manufacturer's instructions. In brief, $0.5 \mu \mathrm{g}$ of plasmid DNA was mixed with Lipofectamine 2000 in a $1 \mu \mathrm{g}: 2 \mu \mathrm{l}$ ratio. Medium was changed $4 \mathrm{~h}$ after transfection to the original cultivation medium and cells were incubated overnight. For transient transfections of HEK293T cells, TransIT293 reagent (Mirus, \#2700) was used according to the manufacturer's instructions. The enhanced green fluorescent protein (EGFP) vector was obtained from Clontech Europe, whereas the pcDNA3-mRuby2 was a gift from Michael Lin (Addgene plasmid \#40260; http://n2t.net/addgene:40260; RRID:Addgene_40260) and pCI-SEP-GluR1 was a gift from Robert Malinow (Addgene plasmid \#24000; http://n2t.net/addgene:24000; RRID:Addgene_24000) (Kopec et al. 2006). EGFP-Rabaptin-5 wild type and EGFP-Rabaptin-5 S407A (Christoforides et al. 2012) were kindly provided by Alex Toker, Beth Israel Deaconess Center, Harvard Medical School, USA. caPKD1-EGFP has already been described elsewhere (Hausser et al. 2002). The GFP-Rabex-5 plasmid (Mattera et al. 2006) was a generous gift from Juan Bonifacino, Division of Intramural Research, NICHD, USA while the pGEX-4 T-2/Rabaptin-5:R5BD was acquired from the lab of Guangpu Li, Fujian Agriculture \& Forestry University, China (Qi et al. 2015). The GFP-Rab5 wild type was received from Lucas Pelkmans, Institute of Molecular Life Sciences, University of Zurich, Switzerland (Nagelkerken et al. 2000).

\section{Immunostaining and confocal laser scanning microscopy}

Hippocampal cultures were fixed on DIV13 for 20 min with 4\% PFA in PBS. After washing with PBS, cells were permeabilized with $0.1 \%$ Triton X-100 in PBS for 5 min. Cells were again washed with PBS and blocked with 5\% FBS and $0.1 \% \mathrm{NaN}_{3}$ in PBS for $1 \mathrm{~h}$ at RT. Subsequently, the corresponding primary antibody (anti-Shank2 (1:2000, Synaptic Systems, \#162204), anti-GluA1 (1:500, Merck, \#MAB2263), anti-EEA1 (1:100, CST, \#3288)) was diluted in blocking buffer and incubated for $1.5 \mathrm{~h}$ at RT. Cells were washed with PBS, and Alexa-Fluor-(546 or 633) labelled secondary antibodies (ThermoFisher Scientific \#A-11030 and \#A-21052, respectively) were diluted 1:500 in blocking buffer and incubated for $1 \mathrm{~h}$ at RT. Finally, samples were mounted in ProLong Gold Antifade. All samples were imaged using a confocal laser scanning microscope (LSM 710, Carl Zeiss) equipped with either a Plan Apochromat 63x/1.40 DIC M27 or an alpha Plan-Apochromat 100x/1.46 Oil DIC objective (Carl Zeiss), using sequential excitation with an $488 \mathrm{~nm}$ Argon laser, an 561 DPSS laser or an $633 \mathrm{~nm}$ $\mathrm{HeNe}$ laser. Image acquisition for the quantitative measurement of GluA1 intensity was done as follows: $\mathrm{z}$-stacks of $0.32 \mu \mathrm{m}$ intervals were acquired throughout the selected neuronal branches of at least $20 \mu \mathrm{m}$ in length. Image processing and analysis were performed with either ZEN blue (Carl Zeiss) or with ImageJ. Regions of interests were selected manually according to clear Shank2 immunopositivity at the plasma membrane along the shaft or within dendritic spines. Mean pixel intensity values of the GluA1 channel within the selected regions of interests were measured, background corrected and normalized to the staining intensity in the control condition. In order to analyze the co-localization between GluA1 and EEA1 signals, single images of selected neuronal branches were acquired. In all experiments, laser power was set so that there would be no saturation and maintained constant throughout the analyses of different samples from the same experiment. Image processing and analysis was performed with ZEN blue (Carl Zeiss) and ImageJ. The Mander's overlap coefficient of GluA1 with EEA1 was calculated and used as a measure of co-localization using the ImageJ plugin JACoP. 


\section{Fluorescence recovery after photobleaching (FRAP)}

212 To investigate AMPAR trafficking to and from the plasma membrane, neuronal cultures were 213 transfected with the super ecliptic pHluorin-tagged GluA1 (SEP-GluA1) and mRuby2 plasmids in a 214 5:3 ratio, and incubated overnight. On the next day, culture medium was changed to pre-heated live 215 cell imaging buffer containing $142 \mathrm{mM} \mathrm{NaCl}, 5.4 \mathrm{Mm} \mathrm{KCl}, 1.8 \mathrm{mM} \mathrm{CaCl}_{2}, 1 \mathrm{mM} \mathrm{NaH}_{2} \mathrm{PO}_{4}, 0.8 \mathrm{mM}$ $216 \mathrm{MgSO}_{4}, 5 \mathrm{mM}$ glucose and $25 \mathrm{mM}$ HEPES with a $\mathrm{pH}$ of 7.4. The diffusion mobility of the GluA1217 containing AMPARs was analyzed at $37^{\circ} \mathrm{C}$ and $5 \% \mathrm{CO}_{2}$ on a Zeiss Axio Observer Spinning Disc 218 Microscope, using an alpha Plan-Apochromat 100x/1.46 Oil DIC objective and a Photometrix Evolve 219512 EMCCD camera for image acquisition. Photobleaching was executed with a UGA-42 firefly 220 photomanipulation system equipped with a $100 \mathrm{~mW} 473 \mathrm{~nm}$ laser (Rapp OptoElectronic, Germany). 221 Cells were initially treated with $2 \mu \mathrm{M}$ of CRT for $10 \mathrm{~min}$, then selected dendritic spines were bleached 222 with a high-intensity laser light (473 nm line, 10\% laser power). Fluorescence intensity in the bleached 223 areas was measured over time both before and after the bleaching event, taking images every 30 seconds for approx. 25 min. Intensity values were corrected with the background intensity values and normalized to the unbleached region. Fitting of the curves was performed with a one-phase exponential equation $Y=Y 0+($ Plateau $-Y 0) *(1-\exp (-K * x))$; Prism 8, GraphPad Software, La Jolla, CA).

\section{MEA recording and analysis}

229 For extracellular voltage recording, hippocampal cell cultures of E17-18 mice embryos were seeded 230 into PLL/laminin coated Axion 24-well BioCircuit (M384-tMEA24W) and 48-well CytoView (M768231 tMEA-48B) MEA plates in a density of $1.5 \times 10^{5}$ and $4 \times 10^{4}$ cells per well, respectively. Five days after 232 plating, half of the culture medium was changed to Brainphys (Stemcell technologies, \#05790) 233 supplemented with SM1 (Stemcell technologies, \#05711), gentamycin (Sigma, \#G1397) and 234 amphotericin B (Gibco, \#15290-026), and treated with cytosin-arabinofuranoside (Sigma, \#C6645). At 12-14 days in vitro, baseline neuronal activity was recorded for 5 minutes by the Maestro Pro recording system (Axion Biosystems) or by a custom-made recording hardware (APPERCELL Biotech Ltd., Hungary), followed by DMSO, CRT and cLTD treatments as described above. 5 minutes long recordings were analysed between 10 to 15 minutes and 90 to 95 minutes following the corresponding treatments.

Action potentials from raw voltage recordings were detected by the Axion AxIS Navigator software or analysed by the NeuroExpress software using a variety of tools including inter-spike time intervals (ISI), burst cycle periods (BCP) as measures of the general intensity of neuronal activity. Bursts were defined as clusters containing at least 3 action potentials with adjacent spikes occurring within $460 \mathrm{~ms}$. Burstiness, a dimensionless parameter was used to describe the overall clustering and separation of burst episodes in the firing pattern. To calculate this parameter, first we identified the smaller of the 2 interburst intervals either preceding or following the actual burst event. Next, we determined the arithmetic mean of the smallest and longest intraburst ISI for the burst. Burstiness was then calculated by dividing the smaller interburst interval by the mean ISI and subtracted 1 from the resulting fraction. Burstiness, therefore, was obtained for each burst event in the spike train. This parameter yields zero

\footnotetext{
${ }^{1}$ ResearchGate. (2018). NeuroExpress: Analysis software for whole-cell electrophysiological data. https://www.researchgate.net/project/NeuroExpress-Analysis-software-for-whole-cell-electrophysiological-data. [Accessed October 26, 2020].
} 
for periodic firing (pacemaker pattern), while increasingly higher values are obtained when the firing becomes more clustered.

\section{Statistical analysis of the data}

For statistical evaluation, unpaired Student's t test, multiple Student's t tests, Mann-Whitney, KruskalWallis and Wilcoxon tests were used employing GraphPad Prism 8 software. Data are displayed as mean \pm SEM, mean $\pm 95 \%$ confidence interval $(\mathrm{CI})$ or Tukey's box-and-whiskers plots.

\section{Ethical approval}

All applicable international, national, and/or institutional guidelines for the care and use of animals were followed. The animal experiments conducted in Stuttgart and Budapest were reviewed and approved by the Regierungspräsidium Stuttgart and by the Animal Ethics Committee of Eötvös Loránd University (approval number: PEI/001/1108-4/2013 and PEI/001/1109-4/2013), respectively. This article does not contain any studies with human participants performed by any of the authors.

\section{Results}

\section{AMPAR basal trafficking is regulated by PKD}

AMPAR surface expression and recycling are highly dynamic, with a half-time in the range of minutes (Henley and Wilkinson 2013). To address the role of PKD in basal AMPAR trafficking, we thus subjected hippocampal neurons to an acute, short-term PKD inhibition (10 min) using CRT0066101 (referred to as CRT), a potent and selective pan-inhibitor of all PKD isoforms (Harikumar et al. 2010). In order to prove that this short-term treatment with CRT effectively inhibits PKD activity, we analyzed autophosphorylation of PKD at serine 916 (pS916PKD) immediately following CRT treatment, as an indicator of kinase activity (Matthews et al. 1999) along with the total levels of PKD (Figure 1a). In line with our previous results (Czondor et al. 2009), we show that endogenous PKD is active in cultured hippocampal neurons under basal conditions. We further detect a strong and significant decrease in the relative level of pS916PKD in CRT treated cells compared to the DMSO treated control (Figure 1a, b), confirming the efficacy of the inhibitor under these conditions.

Next, to assess the surface expression of GluA1-containing AMPARs in hippocampal neurons treated with CRT or the solvent DMSO, we utilized cell surface protein tagging using sulfo-NHS-SS-biotin. After cell lysis, biotinylated proteins were precipitated and the total expression and surface levels of GluA1 were detected by Western Blot analysis (Figure 1c). Our data clearly indicate that already a short-term PKD inhibition significantly increased the relative amount of surface GluA1 compared to the DMSO treatment (Figure 1d).

Under basal conditions, AMPARs have a high turnover within spines (Passafaro et al. 2001, Shi et al. 2001) and are internalized in the vicinity of synapses (Rosendale et al. 2017). To investigate whether short-term PKD inhibition affects the amount of GluA1 within the close vicinity of the synaptic membrane, an antibody feeding assay was performed. Firstly, hippocampal cultures were treated with CRT or DMSO, and subsequently incubated with an antibody detecting the extracellular N-terminal domain of GluA1. Cells were then fixed and stained without permeabilization for the surface-bound $\mathrm{N}$-terminal specific $\alpha$-GluA1 antibody, followed by a subsequent permeabilization and immunostaining for Shank2, a marker of the PSD (Naisbitt et al. 1999) (Figure 1e). Notably, our 
analysis reveals that inhibition of PKD significantly increased GluA1 intensity within Shank2-positive areas compared to the control (Figure 1f). Vice versa, transfection of hippocampal cells with a plasmid encoding a constitutively active S738/742E mutant form of PKD (caPKD-EGFP) significantly decreased the amount of GluA1 in Shank2 positive areas compared to cells transfected with EGFP as control (Figure $1 \mathrm{~g}, \mathrm{~h}$ ).

To measure the impact of PKD inhibition on spontaneous neuronal activity, we cultivated hippocampal neurons on transparent Multi-electrode Array (MEA) plates containing 16 electrodes per well (see Supplementary figure 1a). Neurons at DIV14-15 were treated with DMSO or CRT for 10 min and subsequent extracellular voltage recordings were compared to baseline activity (Figure 1i). Short-term inhibition of PKD by CRT resulted in a significant decrease in interspike intervals compared to pretreatment, basal values (Supplementary figure 1b). Importantly, when the effects of DMSO and CRT treatments were normalized to their corresponding baseline activity, CRT treatment significantly increased firing activity (Figure 1j). In addition, $10 \mathrm{~min}$ CRT treatment significantly increased burstiness compared to basal values (Supplementary figure 1c) or to DMSO treatment (Figure 1k).

Hence, our data provide compelling evidence that PKD activity regulates the amount and PSD localization of GluA1-containing AMPARs in synapses and decreases spontaneous network activity under basal conditions.

\section{PKD controls basal AMPAR endocytosis}

To further investigate how PKD controls AMPAR trafficking, we measured fluorescence recovery after photobleaching (FRAP) of Super Ecliptic pHluorin (SEP)-tagged GluA1. SEP emits fluorescence under neutral $\mathrm{pH}$ conditions, allowing for the study of specific subpopulations of the tagged protein at the cell surface in relation to those fluorochromes which are internalized within vesicles (Kopec et al. 2006). Cultures were co-transfected with mRuby 2 to visualize transfected neuronal cells. Neurons were treated with either CRT or DMSO for $10 \mathrm{~min}$, then the fluorescent signal within the dendritic spines of imaged dendritic branches was bleached and the recovery of the fluorescence intensity was measured for another $10 \mathrm{~min}$ (Figure 2a, b). To calculate the half-life and the mobile fraction of SEP-GluA1, we additionally fitted the recovery curves with a one-phase exponential equation (Figure 2c). In DMSO treated neurons, photobleaching of spine SEP fluorescence to background levels (Figure 2a, b) was followed by a fast recovery $\left(\mathrm{t}^{1} / 2=14.52\right.$ seconds with $95 \%$ CI of $+15.08 \mathrm{CI}$, Figure $\left.2 \mathrm{~d}\right)$ to $61.16 \%$ with a $95 \%$ CI of \pm 3.4 of the original SEP-GluA1 intensity after 183 seconds (Figure 2e). Upon PKD inhibition, GluA1-SEP signal within the spines recovered significantly slower $\left(t^{1} / 2=114.47\right.$ seconds with $95 \%$ CI of 77.67 and 38.26, Figure 2d), albeit to the same level as the control (Fig. 2b, c). Consequently, the mobile fraction of GluA1-SEP did not significantly change between the CRT and DMSO treated cultures (Figure 2e). These results suggest that PKD inhibition slows down the trafficking of cell surface SEP-GluA1.

It is well established that constitutive endocytosis at perisynaptic zones regulates the number of AMPARs at the postsynaptic level (Ehlers 2000, Luscher et al. 1999, Man et al. 2000, Rosendale et al. 2017). Furthermore, under basal conditions, AMPARs undergo a robust time-dependent endocytosis (Ehlers 2000). To clarify whether PKD is involved in basal AMPAR endocytosis, we firstly investigated whether PKD short-term inhibition affects basal trafficking of AMPARs to early endosomes. To that end, neuronal cells expressing EGFP or caPKD-EGFP were treated with CRT or DMSO, fixed, permeabilized and stained for GluA1 and EEA1, a marker of early endosomes (Mu et al. 1995). EEA1 and GluA1 were present on dot-like structures distributed all over the dendritic branch (Figure 2f). This is in line with earlier reports showing that EEA1 participates in the endocytosis of AMPARs and is highly expressed in the postsynaptic compartment of hippocampal synapses (Selak et al. 2006, Selak et al. 2000). To assess whether the amount of GluA1 at early endosomes changes upon 
increased PKD activity, we compared the co-localization of GluA1 and EEA1 by analyzing the Mander's coefficient (Figure 2g) in EGFP or caPKD-EGFP expressing neurons. Our data show that constitutively active PKD significantly enhances the amount of GluA1 at early endosomes. This was rescued back to the control level when cells were treated with CRT for 10 minutes prior to fixation (Figure 2g). Our results thus indicate that enhanced PKD activity promotes AMPAR endocytosis.

To corroborate this finding, we studied the phosphorylation of GluA1 at S831 and S845 depending on PKD activity. S831 phosphorylation is mediated by CaMKII and Protein Kinase C (PKC), promoting the targeting of GluA1 to the PSD and increasing single channel conductance (Barria et al. 1997, Diering et al. 2016, Roche et al. 1996) whereas phosphorylation of S845 is mediated by PKA, leading to an increased single-channel open probability and to targeting or retention of GluA1 at the cell surface (Banke et al. 2000, Diering and Huganir 2018, Man et al. 2007). Cells were treated with CRT or DMSO for $10 \mathrm{~min}$, lysed and both the total and phosphorylated levels of GluA1 were measured via Western Blot (Figure $2 \mathrm{~h}$ and j). Remarkably, while short-term inhibition of PKD did not modify basal pS845 GluA1 levels compared to the control (Figure 2i), our data show a significant increase of basal pS831GluA1 in CRT-treated cells when compared to the control (Figure 2k). These observations are in line with our previous results showing enhanced GluA1 levels at the PSD, slowed down GluA1 cell surface trafficking, and increased spontaneous network activity upon PKD inhibition. As S831phosphorylated GluA1 subunits are enriched at the PSD (Diering et al. 2016), it further suggests that the occurrence of GluA1-containing AMPARs at the PSD is enhanced in PKD-inhibited cells.

Collectively, our data show that PKD activity coordinates the basal endocytic trafficking of GluA1containing AMPARs.

\section{PKD regulates activity-mediated AMPAR endocytosis}

359 Given that PKD activity promotes basal endocytotic AMPAR trafficking, we next investigated whether the kinase also plays a role in agonist-induced AMPAR endocytosis from synapses. To do so, we employed the biotinylation assay of receptor endocytosis using sulfo-NHS-SS-biotin (Ehlers 2000). Briefly, biotinylated hippocampal cultures were treated with CRT or DMSO, followed by 2 minutes of $100 \mu \mathrm{M}$ AMPA-evoked stimulation of AMPARs while simultaneously blocking NMDA receptors with $50 \mu \mathrm{M}$ D-AP5 (see Supplementary figure 2a; treatment is designated as "AMPA"). Cells were incubated additionally for 10 min to allow for endocytosis before the remaining cell surface biotin was cleaved by a glutathione wash and cells were processed for analysis. In this way, GluA1 subunits remain biotinylated only within endosomes. In line with previous results, AMPA treatment induced GluA1 endocytosis as indicated by increased levels of endocytosed GluA1 in comparison to total GluA1 $(126 \% \pm 0.08$ SEM compared to the control, Supplementary figure $2 b, c)$. Strikingly, ligandinduced endocytosis of AMPARs was completely rescued when cells were pre-treated with CRT (72\% \pm 0.13 SEM compared to the control, Supplementary figure 2b, c). Notably, AMPA treatment also enhanced autophosphorylation of PKD, indicating that the kinase is a downstream target of AMPAR signalling. The AMPA-induced increase in kinase activity was completely blocked by pre-treatment of cells with CRT (Supplementary figure $2 \mathrm{~d}, \mathrm{e}$ ). AMPARs thereby evoking LTD (designated as cLTD) (Beattie et al. 2000, Collingridge et al. 2010, Lee et al. 1998, Lee et al. 2002, Lin and Huganir 2007). In order to investigate whether PKD activity is specifically required for LTD induction, cells were pre-treated with CRT or DMSO for 10 min followed by cLTD treatment for additional $5 \mathrm{~min}$. Cells were then changed to an NMDA-free medium and incubated for $15 \mathrm{~min}$ to allow for receptor endocytosis before undergoing surface protein 
biotinylation using sulfo-NHS-SS-biotin (Figure 3a). After cell lysis, biotinylated proteins were precipitated and the total expression and surface levels of GluA1 were detected through Western Blot analysis (Figure 3b). Remarkably, cLTD treatment promoted a significant decrease in surface GluA1 $(78 \% \pm 0.01$ SEM compared to the control) which could be rescued with CRT $(124 \% \pm 0.1$ SEM compared to the control, Figure 3c). Our data also show an enhanced PKD phosphorylation upon cLTD treatment indicating that PKD is activated downstream of NMDA receptors. In line with our previous data (Figure 1), CRT treatment completely prevented NMDA-induced PKD activation (Figure 3d, e). GluA1 dephosphorylation at S845 is a prerequisite for the LTD-induced endocytosis of AMPARs (Diering and Huganir 2018, Lee et al. 1998, Lee et al. 2003). In line with the literature, cLTD treatment promoted a significant decrease in the level of pS845 GluA1 compared to the control. Notably, the NMDA-induced dephosphorylation of S845 was not blocked with CRT treatment (Figure 3f, g) suggesting that PKD acts further downstream.

MEA recordings were additionally taken and compared between control and cLTD treated cultures. Our prior experiments in patch clamp settings have already shown that firing output of such cultures are governed by potent depolarizing events when large numbers of neuron fire synchronously and drive the activity of postsynaptic cells via glutamatergic connections (network bursting) (Rátkai A, Tárnok K, Schlett K, Szücs A. Homeostatic plasticity and burst activity are mediated by hyperpolarizationactivated cation currents and T-type calcium channels in neuronal cultures. Submitted manuscript). In line with the reduced AMPA receptor levels upon cLTD treatment, network activity was significantly decreased, as indicated by elevated interspike interval medians (Supplementary figure 1d). In addition, burstiness was completely diminished upon cLTD (data not shown). This latter observation indicates the reduction of potent depolarizing events, compound excitatory postsynaptic potentials that are the main contributors of action potential emissions of cultured neurons. Importantly, when CRT was present during NMDA treatment and for an additional $10 \mathrm{~min}$, normalized interspike intervals were increased to a significantly lower extent compared to DMSO treated cultures (Figure $3 \mathrm{~h}$ ).

Collectively, our data demonstrate that PKD activity contributes to the endocytosis of AMPARs during normal culture conditions and activity-dependent plasticity.

\section{PKD-mediated phosphorylation of Rabaptin-5 is required for Rab5 activation and activity- mediated AMPAR endocytosis}

The small GTPase Rab5 regulates the fusion kinetics of plasma-derived endocytic vesicles with early endosomes in both basal and activity-mediated trafficking of AMPARs (Bucci et al. 1992, Gorvel et al. 1991, Sziber et al. 2017). Rabaptin-5 forms a complex with Rabex-5, a Rab5-guanosine exchange factor (GEF), and Rab5, thereby promoting Rab5 activation during endocytosis (Horiuchi et al. 1997, Zhang et al. 2014). Notably, Rabaptin-5 is a PKD substrate implicated in the regulation of integrin receptor trafficking in cancer cells (Christoforides et al. 2012). Therefore, we investigated whether PKD-mediated phosphorylation of Rabaptin-5 is required for Rab5 activity. To this end, we performed an effector pulldown assay using HEK293T cells transiently transfected with plasmids encoding EGFP-Rab5 (Rab5) in combination with either EGFP as a control or EGFP-Rabex-5 (Rabex-5) together with EGFP-Rabaptin-5 wild type (Rabaptin-5 wt) or EGFP-Rabaptin-5 S407A (Rabaptin-5 $\mathrm{S} / \mathrm{A}$; Figure S2 f, g). In the latter case, the PKD-specific phosphorylation site of Rabaptin-5 has been exchanged for an alanine, preventing upstream phosphorylation. In line with other reports (Horiuchi et al. 1997, Stenmark et al. 1995, Zhang et al. 2014), we detected an increased level of active Rab5 in cells expressing Rabaptin-5 wt compared to the control cells. By contrast, the phosphodeficient Rabaptin-5 S407A mutant was not able to promote Rab5 activity when compared to control cells 
(Figure S2 f, g). These results demonstrate that PKD-mediated phosphorylation of Rabaptin-5 at serine 407 promotes activation of Rab5.

Additionally, we explored whether phosphorylation of Rabaptin-5 at serine 407 is required to promote synaptic removal of AMPARs upon NMDA-induced LTD. We again employed the GluA1 antibodyfeeding assay on hippocampal cultures transfected with plasmids encoding EGFP, Rabaptin-5 wt or Rabaptin-5 S407A and induced cLTD (Figure $4 \mathrm{a}, \mathrm{b}$ ). When analyzing GluA1 intensity in Shank2 positive areas of untreated cells, we observed a significant increase in the Rabaptin-5 S407A expressing cells in comparison with the EGFP control. This, however, was not the case for the untreated Rabaptin$5 \mathrm{wt}$ expressing cells and clearly indicates that expression of the phosphodeficient mutant of Rabaptin5 increases synaptic GluA1 surface levels under basal conditions. Most importantly, cLTD treatment induced a significant decrease in GluA1 intensity within the synaptic areas both upon EGFP and Rabaptin-5 wt overexpression but not in Rabaptin-5 S407A expressing neurons (Figure 4a, b). Our data thus provide evidence that phosphorylation of Rabaptin-5 at serine 407 regulates basal and activity-induced AMPAR endocytosis most likely downstream of PKD.

\section{Discussion}

In neurons, PKD regulates Golgi function and polarized secretory trafficking thereby contributing to dendrite development and the maintenance of neuronal polarity (Czondor et al. 2009, Horton et al. 2005, Quassollo et al. 2015). Moreover, PKD activity is required for memory formation and learning (Bencsik et al. 2015). However, a role for PKD in the turnover of AMPARs has not been described yet. In the present study, we show that short-term inhibition of PKD activity increases the level of surface and synaptic GluA1-containing AMPARs under basal conditions and during chemically evoked AMPAR internalization. We further demonstrate that PKD is activated downstream of NMDA and AMPA receptor signaling and promotes AMPAR endocytosis, likely through the phosphorylation of the Rab5 effector protein Rabaptin-5.

The dynamic regulation of the number of synaptic AMPARs is one of the main mechanisms modifying long-term synaptic efficacy. Our data highlight PKD as a novel member of the postsynaptic signaling machinery regulating basal and activity-induced endocytosis of AMPARs. This is in line with our previous findings that PKD activity is required for the maintenance of the dendritic tree (Czondor et al. 2009), as well as for the stabilization of the F-actin network within dendritic spines upon induction of LTP and learning and memory formation in vivo (Bencsik et al. 2015). Accordingly, a dual role for PKD in synaptic plasticity becomes apparent: on the one hand, the kinase controls the activity-induced potentiation and enlargement of spines during chemically-induced LTP through stabilization of the Factin network within the newly enlarged dendritic spines (Bencsik et al. 2015), while on the other hand it is required for the elimination of AMPARs from the cell surface during cLTD through phosphorylation of Rabaptin-5, and the subsequent activation of Rab5.

In line with our results, PKD has been already described to participate in endocytosis and recycling of membrane proteins in non-neuronal cells. In fibroblasts, PKD activity is necessary for PDGF-driven short-loop recycling of $\alpha_{v} \beta_{3}$ integrin (Woods et al. 2004). However, in neurons, expression of dominant-negative kinase-dead PKD (kdPKD) was reported to increase the endocytosis rate of the TfR and LRP dendritic membrane proteins (Bisbal et al. 2008). At first glimpse, these data seem to contradict our findings, however, the different experimental approaches used to block PKD activity (one day of kdPKD expression vs acute CRT-mediated inhibition) may account for the discrepancies 
observed. This is also underpinned by the fact that expression of kdPKD1 affected dendrite development and neuronal polarity (Czondor et al. 2009) whereas we could not observe any morphological changes upon short-term PKD inhibition (data not shown). Furthermore, due to its established role in polarized secretory trafficking in neurons, long-term inhibition of PKD activity is expected to affect exocytotic trafficking as well (Horton et al. 2005). Thus, the short-term chemical inhibition used in this study is more suitable to analyze PKD-dependent fast changes in endocytosis.

We further found that phosphorylation of the PKD substrate Rabaptin-5 regulates synaptic AMPAR surface expression. Rabaptin-5 and the Rab5-GEF Rabex-5 function together to activate Rab5 and to promote the fusion of endocytotic vesicles with early endosomes (Horiuchi et al. 1997, Stenmark et al. 1995, Zhang et al. 2014). Rab5 plays a critical role in the regulation of constitutive and activitydependent AMPAR endocytosis (Brown et al. 2005, Glebov et al. 2015) and is essential for LTD (Brown et al. 2005). By investigating another potential downstream target of PKD, the Rab5-GEF RIN1, we recently showed that enhancing Rab5 activity is a critical step during activity-dependent AMPAR internalization (Sziber et al. 2017). In accordance, loss or inhibition of the Rab5 effector protein EEA1 results in increased GluA1-containing AMPAR surface expression (Selak et al. 2006, $\mathrm{Xu}$ and Pozzo-Miller 2017) reminiscent of the increased GluA1 surface expression observed upon short-term PKD inhibition. Additionally, expression of constitutive active PKD enhanced the colocalization of GluA1 with EEA1 further supporting a role for PKD in promoting AMPAR endocytosis through Rabaptin-5 and Rab5.

Notably, Rabaptin-5 is also an effector of Rab4 (Vitale et al. 1998), which regulates cargo recycling (van der Sluijs et al. 1992) and is also found on early endosomes (Ehlers 2000, Sonnichsen et al. 2000). In neurons, Rab4 regulates dendritic spine maintenance and constitutive AMPAR recycling from sorting endosomes (Brown et al. 2007, Gu et al. 2016). Interestingly, Rab4 and Rabex-5 recruit Rabaptin-5 to early endosomes to subsequently activate Rab5 (Kalin et al. 2015). Additionally, in breast cancer cells, PKD-mediated Rabaptin-5 phosphorylation controls $\alpha_{v} \beta_{3}$ integrin recycling from early endosomes in a Rab4-dependent manner (Christoforides et al. 2012, Woods et al. 2004). Our results suggest that PKD-mediated phosphorylation of Rabaptin-5 promotes AMPAR endocytosis through coordinating its interaction with Rab4 and activation of Rab5. In support of this are the findings i) that Rabaptin-5 S407A cannot properly activate Rab5 (this study) and ii) that its ability to interact with Rab4 is markedly reduced (Christoforides et al. 2012).

The presence and regulation of post-translational modifications on neurotransmitter receptors modifies their function and trafficking and thus synaptic strength. Post-translational modifications have been described to mediate numerous processes including AMPAR membrane targeting, retention, conductance, endocytosis and degradation (Boehm et al. 2006, Chung et al. 2000, Coultrap et al. 2014, Esteban et al. 2003, Hayashi and Huganir 2004, Lee et al. 2010, Roche et al. 1996, Seidenman et al. 2003, Steinberg et al. 2006, Widagdo et al. 2015). Several phosphorylation sites on GluA1 have already been shown to be important for stability at the PSD and AMPAR conductance (Boehm et al. 2006, Derkach et al. 1999, Esteban 2003, Roche et al. 1996), with S831 and S845 being best understood. CaMKII/PKC-mediated phosphorylation of S831 increases single-channel conductance (Barria et al. 1997, Derkach et al. 1999). Moreover, under basal conditions, pS831-GluA1 receptors are enriched at the PSD suggesting that phosphorylation at S831 increases GluA1 targeting to this compartment (Diering et al. 2016). In agreement with this, we detected an increase in pS831 GluA1 levels upon short-term PKD inhibition, presumably as a consequence of impaired AMPAR endocytosis. PKAmediated phosphorylation of S845 has been described to promote GluA1 targeting to the cell surface and single-channel open probability (Banke et al. 2000, Lee et al. 2010, Man et al. 2007). Notably, dephosphorylation at S845 is required for AMPAR endocytosis upon LTD (Lee et al. 2010). Our data 
show that acute CRT treatment did not rescue the dephosphorylation of GluA1 at serine 845 in response to cLTD. This suggests that i) PKD activity promotes AMPAR endocytosis downstream of S845 dephosphorylation and ii) upon PKD inhibition, AMPARs are primed for their endocytosis but remain on the surface.

518 Based on our data we propose the following model on how PKD regulates AMPAR endocytic trafficking: under basal conditions, endogenous PKD is active and phosphorylates Rabaptin-5 thereby promoting its interaction with Rab4 on early endosomes and the activation of Rab5 through Rabex -5 . Consequently, AMPARs are taken back from the synaptic membrane by Rab5-dependent endocytosis. Chemically-induced AMPAR endocytosis either through NMDA receptor or AMPAR stimulation further enhances PKD activity thus accelerating AMPAR elimination from the surface (Figure 4c).

As most excitatory transmission in the brain is mediated by the AMPARs, their dysfunction has been observed in many neuronal disorders, with Alzheimer disease (AD) being the best reported so far. High concentration of soluble oligomeric A $\beta$ induces the removal of surface AMPARs at synapses, leading to synaptic depression and impaired synaptic plasticity and memory (Hsieh et al. 2006, Shankar et al. 2007). Strikingly, at preclinical stages of $\mathrm{AD}$, the appearance of enlarged Rab5-positive early endosomes was associated with elevated Rab4 levels and Rabaptin-5 translocation to endosomes (Cataldo et al. 2000). Indeed, hyperactivation of Rab5 has been observed in AD patients and mouse models of AD disease (Ginsberg, Alldred, et al. 2010, Ginsberg et al. 2011, Ginsberg, Mufson, et al. 2010). These data imply that endocytic uptake of AMPARs is enhanced in AD, however, whether this also correlates with increased PKD activity needs to be addressed in future studies.

\section{Acknowledgements}

We are grateful to Viktória Szentgyörgyi and Gisela Link (Institute for Cell Biology and Immunology, Germany) for their excellent technical assistance and Raluca Tamas (Institute for Cell Biology and Immunology, Germany) for critical reading of the manuscript.

\section{Conflicts of interest}

The authors declare that the research was conducted in the absence of any commercial or financial relationships that could be construed as a potential conflict of interest.

\section{Authors' contributions}

Conceptualization: Angelika Hausser, Katalin Schlett; Methodology: Carlos O. Oueslati Morales, Attila Ignacz, Norbert Bencsik, Aniko E. Ratkai, Wolfgang S. Lieb; Formal analysis and investigation: Carlos O. Oueslati Morales, Attila Ignacz, Attila Szücs, Aniko E. Ratkai, Norbert Bencsik, Stephan A. Eisler, Wolfgang S. Lieb; Writing - original draft preparation: Carlos O. Oueslati Morales; Writing - review and editing: Angelika Hausser, Katalin Schlett; Funding acquisition: Angelika Hausser, Katalin Schlett. All authors contributed to the article and approved the submitted version. 


\section{Funding}

This study was funded by a grant from the German Research Foundation to AH (DFG HA-357/11-2), a travel exchange program funded by the DAAD (PPP Hungary 57215775 and 57392635), and by the National Brain Research Programs (KTIA_NAP_13-2-2014-0018 and 2017-1.2.1-NKP-2017-00002) to KS as well as the VEKOP-2.3.3-15-2016-00007 grant from NRDIO.

\section{Bibliography}

Banke TG, Bowie D, Lee H, Huganir RL, Schousboe A, Traynelis SF. 2000. Control of GluR1 AMPA receptor function by cAMP-dependent protein kinase. The Journal of neuroscience : the official journal of the Society for Neuroscience. Jan 1;20:89-102.

Barria A, Derkach V, Soderling T. 1997. Identification of the Ca2+/calmodulin-dependent protein kinase II regulatory phosphorylation site in the alpha-amino-3-hydroxyl-5-methyl-4-isoxazolepropionate-type glutamate receptor. The Journal of biological chemistry. Dec 26;272:32727-32730.

Beattie EC, Carroll RC, Yu X, Morishita W, Yasuda H, von Zastrow M, Malenka RC. 2000. Regulation of AMPA receptor endocytosis by a signaling mechanism shared with LTD. Nature neuroscience. Dec;3:1291-1300.

Bencsik N, Sziber Z, Liliom H, Tarnok K, Borbely S, Gulyas M, Ratkai A, Szucs A, Hazai-Novak D, Ellwanger K, et al. 2015. Protein kinase D promotes plasticity-induced F-actin stabilization in dendritic spines and regulates memory formation. The Journal of cell biology. Aug 31;210:771-783.

Bisbal M, Conde C, Donoso M, Bollati F, Sesma J, Quiroga S, Diaz Anel A, Malhotra V, Marzolo MP, Caceres A. 2008. Protein kinase d regulates trafficking of dendritic membrane proteins in developing neurons. The Journal of neuroscience : the official journal of the Society for Neuroscience. Sep 10;28:9297-9308.

Blanpied TA, Scott DB, Ehlers MD. 2002. Dynamics and regulation of clathrin coats at specialized endocytic zones of dendrites and spines. Neuron. Oct 24;36:435-449.

Boehm J, Kang MG, Johnson RC, Esteban J, Huganir RL, Malinow R. 2006. Synaptic incorporation of AMPA receptors during LTP is controlled by a PKC phosphorylation site on GluR1. Neuron. Jul 20;51:213-225.

Brown TC, Correia SS, Petrok CN, Esteban JA. 2007. Functional compartmentalization of endosomal trafficking for the synaptic delivery of AMPA receptors during long-term potentiation. The Journal of neuroscience : the official journal of the Society for Neuroscience. Nov 28;27:1331113315.

Brown TC, Tran IC, Backos DS, Esteban JA. 2005. NMDA receptor-dependent activation of the small GTPase Rab5 drives the removal of synaptic AMPA receptors during hippocampal LTD. Neuron. Jan 6;45:81-94.

Bucci C, Parton RG, Mather IH, Stunnenberg H, Simons K, Hoflack B, Zerial M. 1992. The small GTPase rab5 functions as a regulatory factor in the early endocytic pathway. Cell. Sep 4;70:715-728.

Carroll RC, Lissin DV, von Zastrow M, Nicoll RA, Malenka RC. 1999. Rapid redistribution of glutamate receptors contributes to long-term depression in hippocampal cultures. Nature neuroscience. May;2:454-460. 
Cataldo AM, Peterhoff CM, Troncoso JC, Gomez-Isla T, Hyman BT, Nixon RA. 2000. Endocytic pathway abnormalities precede amyloid beta deposition in sporadic Alzheimer's disease and Down syndrome: differential effects of APOE genotype and presenilin mutations. The American journal of pathology. Jul;157:277-286.

Chater TE, Goda Y. 2014. The role of AMPA receptors in postsynaptic mechanisms of synaptic plasticity. Front Cell Neurosci.8:401.

Christoforides C, Rainero E, Brown KK, Norman JC, Toker A. 2012. PKD controls alphavbeta3 integrin recycling and tumor cell invasive migration through its substrate Rabaptin-5. Developmental cell. Sep 11;23:560-572.

Chung HJ, Xia J, Scannevin RH, Zhang X, Huganir RL. 2000. Phosphorylation of the AMPA receptor subunit GluR2 differentially regulates its interaction with PDZ domain-containing proteins. The Journal of neuroscience : the official journal of the Society for Neuroscience. Oct 1;20:72587267.

Collingridge GL, Peineau S, Howland JG, Wang YT. 2010. Long-term depression in the CNS. Nature reviews Neuroscience. Jul;11:459-473.

Coultrap SJ, Freund RK, O'Leary H, Sanderson JL, Roche KW, Dell'Acqua ML, Bayer KU. 2014. Autonomous CaMKII mediates both LTP and LTD using a mechanism for differential substrate site selection. Cell reports. Feb 13;6:431-437.

Czondor K, Ellwanger K, Fuchs YF, Lutz S, Gulyas M, Mansuy IM, Hausser A, Pfizenmaier K, Schlett K. 2009. Protein kinase D controls the integrity of Golgi apparatus and the maintenance of dendritic arborization in hippocampal neurons. Molecular biology of the cell. Apr;20:2108-2120.

Derkach V, Barria A, Soderling TR. 1999. Ca2+/calmodulin-kinase II enhances channel conductance of alpha-amino-3-hydroxy-5-methyl-4-isoxazolepropionate type glutamate receptors. Proceedings of the National Academy of Sciences of the United States of America. Mar 16;96:3269-3274.

Diering GH, Heo S, Hussain NK, Liu B, Huganir RL. 2016. Extensive phosphorylation of AMPA receptors in neurons. Proceedings of the National Academy of Sciences of the United States of America. Aug 16;113:E4920-4927.

Diering GH, Huganir RL. 2018. The AMPA Receptor Code of Synaptic Plasticity. Neuron. Oct 24;100:314-329.

Ehlers MD. 2000. Reinsertion or degradation of AMPA receptors determined by activity-dependent endocytic sorting. Neuron. Nov;28:511-525.

Ellwanger K, Hausser A. 2013. Physiological functions of protein kinase D in vivo. IUBMB life. Feb;65:98-107.

Esteban JA. 2003. AMPA receptor trafficking: a road map for synaptic plasticity. Molecular interventions. Oct;3:375-385.

Esteban JA, Shi SH, Wilson C, Nuriya M, Huganir RL, Malinow R. 2003. PKA phosphorylation of AMPA receptor subunits controls synaptic trafficking underlying plasticity. Nature neuroscience. Feb;6:136-143.

Ginsberg SD, Alldred MJ, Counts SE, Cataldo AM, Neve RL, Jiang Y, Wuu J, Chao MV, Mufson EJ, Nixon RA, et al. 2010. Microarray analysis of hippocampal CA1 neurons implicates early endosomal dysfunction during Alzheimer's disease progression. Biological psychiatry. Nov $15 ; 68: 885-893$. 
Ginsberg SD, Mufson EJ, Alldred MJ, Counts SE, Wuu J, Nixon RA, Che S. 2011. Upregulation of select rab GTPases in cholinergic basal forebrain neurons in mild cognitive impairment and Alzheimer's disease. Journal of chemical neuroanatomy. Oct;42:102-110.

638 Ginsberg SD, Mufson EJ, Counts SE, Wuu J, Alldred MJ, Nixon RA, Che S. 2010. Regional selectivity of rab5 and rab7 protein upregulation in mild cognitive impairment and Alzheimer's disease. Journal of Alzheimer's disease : JAD.22:631-639.

Glebov OO, Tigaret CM, Mellor JR, Henley JM. 2015. Clathrin-independent trafficking of AMPA receptors. The Journal of neuroscience : the official journal of the Society for Neuroscience. Mar $643 \quad 25 ; 35: 4830-4836$.

644 Gorvel JP, Chavrier P, Zerial M, Gruenberg J. 1991. rab5 controls early endosome fusion in vitro. 645 Cell. Mar 8;64:915-925.

646 Greger IH, Khatri L, Ziff EB. 2002. RNA editing at arg607 controls AMPA receptor exit from the 647 endoplasmic reticulum. Neuron. May 30;34:759-772.

$648 \mathrm{Gu}$ Y, Chiu SL, Liu B, Wu PH, Delannoy M, Lin DT, Wirtz D, Huganir RL. 2016. Differential 649 vesicular sorting of AMPA and GABAA receptors. Proceedings of the National Academy of 650 Sciences of the United States of America. Feb 16;113:E922-931.

651 Harikumar KB, Kunnumakkara AB, Ochi N, Tong Z, Deorukhkar A, Sung B, Kelland L, Jamieson S, 652 Sutherland R, Raynham T, et al. 2010. A novel small-molecule inhibitor of protein kinase D blocks 653 pancreatic cancer growth in vitro and in vivo. Molecular cancer therapeutics. May;9:1136-1146.

654 Hausser A, Link G, Bamberg L, Burzlaff A, Lutz S, Pfizenmaier K, Johannes FJ. 2002. Structural 655 requirements for localization and activation of protein kinase $\mathrm{C} \mathrm{mu}(\mathrm{PKC} \mathrm{mu})$ at the Golgi 656 compartment. The Journal of cell biology. Jan 7;156:65-74.

657 Hayashi T, Huganir RL. 2004. Tyrosine phosphorylation and regulation of the AMPA receptor by 658 SRC family tyrosine kinases. The Journal of neuroscience : the official journal of the Society for 659 Neuroscience. Jul 7;24:6152-6160.

660 Hayashi Y, Shi SH, Esteban JA, Piccini A, Poncer JC, Malinow R. 2000. Driving AMPA receptors 661 into synapses by LTP and CaMKII: requirement for GluR1 and PDZ domain interaction. Science. 662 Mar 24;287:2262-2267.

663 Henley JM, Wilkinson KA. 2013. AMPA receptor trafficking and the mechanisms underlying 664 synaptic plasticity and cognitive aging. Dialogues in clinical neuroscience. Mar;15:11-27.

665 Horiuchi H, Lippe R, McBride HM, Rubino M, Woodman P, Stenmark H, Rybin V, Wilm M, 666 Ashman K, Mann M, et al. 1997. A novel Rab5 GDP/GTP exchange factor complexed to Rabaptin-5 667 links nucleotide exchange to effector recruitment and function. Cell. Sep 19;90:1149-1159.

668 Horton AC, Racz B, Monson EE, Lin AL, Weinberg RJ, Ehlers MD. 2005. Polarized secretory 669 trafficking directs cargo for asymmetric dendrite growth and morphogenesis. Neuron. Dec 8;48:757670771.

671 Hsieh H, Boehm J, Sato C, Iwatsubo T, Tomita T, Sisodia S, Malinow R. 2006. AMPAR removal 672 underlies Abeta-induced synaptic depression and dendritic spine loss. Neuron. Dec 7;52:831-843.

673 Huganir RL, Nicoll RA. 2013. AMPARs and synaptic plasticity: the last 25 years. Neuron. Oct $67430 ; 80: 704-717$.

675 Kalin S, Hirschmann DT, Buser DP, Spiess M. 2015. Rabaptin5 is recruited to endosomes by Rab4 676 and Rabex5 to regulate endosome maturation. Journal of cell science. Nov 15;128:4126-4137. 
Kopec CD, Li B, Wei W, Boehm J, Malinow R. 2006. Glutamate receptor exocytosis and spine enlargement during chemically induced long-term potentiation. The Journal of neuroscience : the official journal of the Society for Neuroscience. Feb 15;26:2000-2009.

680 Lee HK, Kameyama K, Huganir RL, Bear MF. 1998. NMDA induces long-term synaptic depression and dephosphorylation of the GluR1 subunit of AMPA receptors in hippocampus. Neuron.

682 Nov;21:1151-1162.

683 Lee HK, Takamiya K, Han JS, Man H, Kim CH, Rumbaugh G, Yu S, Ding L, He C, Petralia RS, et 684 al. 2003. Phosphorylation of the AMPA receptor GluR1 subunit is required for synaptic plasticity and 685 retention of spatial memory. Cell. Mar 7;112:631-643.

686 Lee HK, Takamiya K, He K, Song L, Huganir RL. 2010. Specific roles of AMPA receptor subunit 687 GluR1 (GluA1) phosphorylation sites in regulating synaptic plasticity in the CA1 region of 688 hippocampus. Journal of neurophysiology. Jan;103:479-489.

689 Lee SH, Liu L, Wang YT, Sheng M. 2002. Clathrin adaptor AP2 and NSF interact with overlapping 690 sites of GluR2 and play distinct roles in AMPA receptor trafficking and hippocampal LTD. Neuron. 691 Nov 14;36:661-674.

692 Lin DT, Huganir RL. 2007. PICK1 and phosphorylation of the glutamate receptor 2 (GluR2) AMPA 693 receptor subunit regulates GluR2 recycling after NMDA receptor-induced internalization. The 694 Journal of neuroscience : the official journal of the Society for Neuroscience. Dec 12;27:1390369513908.

696 Lu W, Shi Y, Jackson AC, Bjorgan K, During MJ, Sprengel R, Seeburg PH, Nicoll RA. 2009. 697 Subunit composition of synaptic AMPA receptors revealed by a single-cell genetic approach. 698 Neuron. Apr 30;62:254-268.

699 Luscher C, Xia H, Beattie EC, Carroll RC, von Zastrow M, Malenka RC, Nicoll RA. 1999. Role of 700 AMPA receptor cycling in synaptic transmission and plasticity. Neuron. Nov;24:649-658.

701 Makino H, Malinow R. 2009. AMPA receptor incorporation into synapses during LTP: the role of 702 lateral movement and exocytosis. Neuron. Nov 12;64:381-390.

703 Malinow R, Malenka RC. 2002. AMPA receptor trafficking and synaptic plasticity. Annual review of 704 neuroscience.25:103-126.

705 Man HY, Lin JW, Ju WH, Ahmadian G, Liu L, Becker LE, Sheng M, Wang YT. 2000. Regulation of 706 AMPA receptor-mediated synaptic transmission by clathrin-dependent receptor internalization.

707 Neuron. Mar;25:649-662.

708 Man HY, Sekine-Aizawa Y, Huganir RL. 2007. Regulation of \{alpha\}-amino-3-hydroxy-5-methyl-4709 isoxazolepropionic acid receptor trafficking through PKA phosphorylation of the Glu receptor 1 710 subunit. Proceedings of the National Academy of Sciences of the United States of America. Feb $711 \quad 27 ; 104: 3579-3584$.

712 Mattera R, Tsai YC, Weissman AM, Bonifacino JS. 2006. The Rab5 guanine nucleotide exchange 713 factor Rabex-5 binds ubiquitin (Ub) and functions as a Ub ligase through an atypical Ub-interacting 714 motif and a zinc finger domain. The Journal of biological chemistry. Mar 10;281:6874-6883.

715 Matthews SA, Rozengurt E, Cantrell D. 1999. Characterization of serine 916 as an in vivo 716 autophosphorylation site for protein kinase D/Protein kinase Cmu. The Journal of biological 717 chemistry. Sep 10;274:26543-26549.

718 Mu FT, Callaghan JM, Steele-Mortimer O, Stenmark H, Parton RG, Campbell PL, McCluskey J, 719 Yeo JP, Tock EP, Toh BH. 1995. EEA1, an early endosome-associated protein. EEA1 is a conserved 
alpha-helical peripheral membrane protein flanked by cysteine "fingers" and contains a calmodulinbinding IQ motif. The Journal of biological chemistry. Jun 2;270:13503-13511.

Nagelkerken B, Van Anken E, Van Raak M, Gerez L, Mohrmann K, Van Uden N, Holthuizen J, Pelkmans L, Van Der Sluijs P. 2000. Rabaptin4, a novel effector of the small GTPase rab4a, is recruited to perinuclear recycling vesicles. The Biochemical journal. Mar 15;346 Pt 3:593-601.

Naisbitt S, Kim E, Tu JC, Xiao B, Sala C, Valtschanoff J, Weinberg RJ, Worley PF, Sheng M. 1999. Shank, a novel family of postsynaptic density proteins that binds to the NMDA receptor/PSD95/GKAP complex and cortactin. Neuron. Jul;23:569-582.

Olayioye MA, Barisic S, Hausser A. 2013. Multi-level control of actin dynamics by protein kinase D. Cellular signalling. Sep;25:1739-1747.

Oster H, Abraham D, Leitges M. 2006. Expression of the protein kinase D (PKD) family during mouse embryogenesis. Gene expression patterns : GEP. Apr;6:400-408.

Passafaro M, Piech V, Sheng M. 2001. Subunit-specific temporal and spatial patterns of AMPA receptor exocytosis in hippocampal neurons. Nature neuroscience. Sep;4:917-926.

Pick JE, Ziff EB. 2018. Regulation of AMPA receptor trafficking and exit from the endoplasmic reticulum. Molecular and cellular neurosciences. Sep;91:3-9.

Qi Y, Liang Z, Wang Z, Lu G, Li G. 2015. Determination of Rab5 activity in the cell by effector pull-down assay. Methods in molecular biology.1298:259-270.

Quassollo G, Wojnacki J, Salas DA, Gastaldi L, Marzolo MP, Conde C, Bisbal M, Couve A, Caceres A. 2015. A RhoA Signaling Pathway Regulates Dendritic Golgi Outpost Formation. Current biology : CB. Apr 20;25:971-982.

Reinhardt R, Truebestein L, Schmidt HA, Leonard TA. 2020. It Takes Two to Tango: Activation of Protein Kinase D by Dimerization. BioEssays : news and reviews in molecular, cellular and developmental biology. Apr;42:e1900222.

Roche KW, O'Brien RJ, Mammen AL, Bernhardt J, Huganir RL. 1996. Characterization of multiple phosphorylation sites on the AMPA receptor GluR1 subunit. Neuron. Jun;16:1179-1188.

Rosendale M, Jullie D, Choquet D, Perrais D. 2017. Spatial and Temporal Regulation of Receptor Endocytosis in Neuronal Dendrites Revealed by Imaging of Single Vesicle Formation. Cell reports. Feb 21;18:1840-1847.

Seidenman KJ, Steinberg JP, Huganir R, Malinow R. 2003. Glutamate receptor subunit 2 Serine 880 phosphorylation modulates synaptic transmission and mediates plasticity in CA1 pyramidal cells. The Journal of neuroscience : the official journal of the Society for Neuroscience. Oct 8;23:92209228.

Selak S, Paternain AV, Fritzler MJ, Lerma J. 2006. Human autoantibodies against early endosome antigen-1 enhance excitatory synaptic transmission. Neuroscience. Dec 28;143:953-964.

Selak S, Woodman RC, Fritzler MJ. 2000. Autoantibodies to early endosome antigen (EEA1) produce a staining pattern resembling cytoplasmic anti-neutrophil cytoplasmic antibodies (CANCA). Clinical and experimental immunology. Dec;122:493-498.

Shankar GM, Bloodgood BL, Townsend M, Walsh DM, Selkoe DJ, Sabatini BL. 2007. Natural oligomers of the Alzheimer amyloid-beta protein induce reversible synapse loss by modulating an NMDA-type glutamate receptor-dependent signaling pathway. The Journal of neuroscience : the official journal of the Society for Neuroscience. Mar 14;27:2866-2875. 
Shi S, Hayashi Y, Esteban JA, Malinow R. 2001. Subunit-specific rules governing AMPA receptor trafficking to synapses in hippocampal pyramidal neurons. Cell. May 4;105:331-343.

Shi SH, Hayashi Y, Petralia RS, Zaman SH, Wenthold RJ, Svoboda K, Malinow R. 1999. Rapid spine delivery and redistribution of AMPA receptors after synaptic NMDA receptor activation. Science. Jun 11;284:1811-1816.

Sonnichsen B, De Renzis S, Nielsen E, Rietdorf J, Zerial M. 2000. Distinct membrane domains on endosomes in the recycling pathway visualized by multicolor imaging of Rab4, Rab5, and Rab11. The Journal of cell biology. May 15;149:901-914.

Steinberg JP, Takamiya K, Shen Y, Xia J, Rubio ME, Yu S, Jin W, Thomas GM, Linden DJ, Huganir RL. 2006. Targeted in vivo mutations of the AMPA receptor subunit GluR2 and its interacting protein PICK1 eliminate cerebellar long-term depression. Neuron. Mar 16;49:845-860.

Stenmark H, Vitale G, Ullrich O, Zerial M. 1995. Rabaptin-5 is a direct effector of the small GTPase Rab5 in endocytic membrane fusion. Cell. Nov 3;83:423-432.

Sziber Z, Liliom H, Morales CO, Ignacz A, Ratkai AE, Ellwanger K, Link G, Szucs A, Hausser A, Schlett K. 2017. Ras and Rab interactor 1 controls neuronal plasticity by coordinating dendritic filopodial motility and AMPA receptor turnover. Molecular biology of the cell. Jan 15;28:285-295.

van der Sluijs P, Hoogenraad CC. 2011. New insights in endosomal dynamics and AMPA receptor trafficking. Seminars in cell \& developmental biology. Jul;22:499-505.

van der Sluijs P, Hull M, Webster P, Male P, Goud B, Mellman I. 1992. The small GTP-binding protein rab4 controls an early sorting event on the endocytic pathway. Cell. Sep 4;70:729-740.

Vitale G, Rybin V, Christoforidis S, Thornqvist P, McCaffrey M, Stenmark H, Zerial M. 1998. Distinct Rab-binding domains mediate the interaction of Rabaptin-5 with GTP-bound Rab4 and Rab5. The EMBO journal. Apr 1;17:1941-1951.

Widagdo J, Chai YJ, Ridder MC, Chau YQ, Johnson RC, Sah P, Huganir RL, Anggono V. 2015. Activity-Dependent Ubiquitination of GluA1 and GluA2 Regulates AMPA Receptor Intracellular Sorting and Degradation. Cell reports. Feb 10;10:783-795.

Woods AJ, White DP, Caswell PT, Norman JC. 2004. PKD1/PKCmu promotes alphavbeta3 integrin recycling and delivery to nascent focal adhesions. The EMBO journal. Jul 7;23:2531-2543.

Xu X, Pozzo-Miller L. 2017. EEA1 restores homeostatic synaptic plasticity in hippocampal neurons from Rett syndrome mice. The Journal of physiology. Aug 15;595:5699-5712.

Yin DM, Huang YH, Zhu YB, Wang Y. 2008. Both the establishment and maintenance of neuronal polarity require the activity of protein kinase $\mathrm{D}$ in the Golgi apparatus. The Journal of neuroscience : the official journal of the Society for Neuroscience. Aug 27;28:8832-8843.

Zhang Z, Zhang T, Wang S, Gong Z, Tang C, Chen J, Ding J. 2014. Molecular mechanism for Rabex-5 GEF activation by Rabaptin-5. eLife. Jun 23;3.

Zhu JJ, Esteban JA, Hayashi Y, Malinow R. 2000. Postnatal synaptic potentiation: delivery of GluR4-containing AMPA receptors by spontaneous activity. Nature neuroscience. Nov;3:1098-1106. 


\section{Figure legends}

\section{Fig. 1 PKD regulates basal AMPAR trafficking}

804 Representative Western Blots obtained from hippocampal cultures treated with DMSO (-) or CRT displaying PKD and pS916PKD levels (a) and total and surface GluA1 levels upon biotinylation (c). Actin served as a loading control and is absent from the precipitated samples. (b and d) Quantification of data shown in (a) and (c), respectively, using densitometry analysis. Data from CRT-treated cultures are presented as mean line density \pm SEM normalized to the control cultures; each dot represents one independent experiment. Statistical comparison was performed by unpaired two-tailed t-test. The dotted line indicates the control level. (e and g) Representative inverted single-channel and colored merged pictures of neuronal dendritic branches stained for the extracellular N-terminal domain of GluA1 and the PSD marker Shank2. Arrows point to Shank2-positive areas included in the analysis. The EGFP signal is not included in the colored merged image. (f and h) Quantification of pictures shown in (e) and (g), respectively. Data were normalized for the DMSO (f) or EGFP control (h). The boxplots show the results of three independent experiments. Center lines show the medians; box limits indicate the $25^{\text {th }}$ and $75^{\text {th }}$ percentiles; whiskers extend 1.5 times the interquartile range from the $25^{\text {th }}$ and $75^{\text {th }}$ percentiles, outliers are represented by dots. The number of investigated sample points is $\mathrm{N}=171$ and 273 (f) or $\mathrm{N}=220$ and 223 (h), respectively. The significance of differences was analyzed by a Mann-Whitney test (two-tailed). (i) Short segments of raw voltage traces before and after CRT treatment. ( $\mathbf{j}$ and $\mathbf{k}$ ) Quantification of data shown in (i). The interspike interval median (j) and the burstiness median $(\mathrm{k})$ were normalized for the DMSO controls. The boxplots show the results of three independent experiments. Center lines show the medians; box limits indicate the $25^{\text {th }}$ and $75^{\text {th }}$ percentiles; whiskers extend 1.5 times the interquartile range from the $25^{\text {th }}$ and $75^{\text {th }}$ percentiles, outliers are represented by dots. The significance of differences was analyzed by a Mann-Whitney test (twotailed). ${ }^{\mathrm{p}}<0.05, * * \mathrm{p}<0.01, * * * \mathrm{p}<0.001, * * * * \mathrm{p}<0.0001$. Scale bar $1 \mu \mathrm{m}$

\section{Fig. 2 PKD specifically controls AMPAR endocytosis}

(a) Representative pictures of neuronal dendritic spines expressing Super Ecliptic pHluorin-tagged GluA1 (SEP-GluA1) and mRuby2. Cells were treated with DMSO as a control or CRT prior to the bleaching event. Images show dendritic spines before bleaching, as well as 5, 60 and 600s afterwards. (b) Fluorescence recovery curve and (c) one-phase association curve fitting of the SEP-GluA1 intensity after bleaching. Data shown as mean \pm SEM (b) or with 95\% CI (c); n=3-4, N=14 and 27 analyzed cells, for DMSO and CRT-treated conditions, respectively. The significance of differences was analyzed by a multiple t-test, using the Holm-Sidak method. (d) Recovery half-time of the SEP-GluA1 signal and (e) mobile GluA1 fraction. Data shown as mean $\pm 95 \%$ CI. (f) Representative pictures of neuronal dendritic branches expressing EGFP or caPKD-EGFP. Cells were treated with DMSO (-) or CRT, fixed and stained for the early endosome marker EEA1 and for GluA1. Arrows point to the EEA1-positive early endosomes included in the analysis. The EGFP signal is not included in the colored merged image. (g) Manders' overlap coefficient for EEA1 and GluA1. Quantification of pictures shown in (f). The boxplot shows the results of three independent experiments. Center lines show the medians; box limits indicate the $25^{\text {th }}$ and $75^{\text {th }}$ percentiles; whiskers extend 1.5 times the interquartile range from the $25^{\text {th }}$ and $75^{\text {th }}$ percentiles, outliers are represented by dots; $\mathrm{N}=28$ sample points each. The significance of differences was analyzed by a Kruskal-Wallis test with Dunn's multiple correction. Representative Western Blot displaying total and pS845 GluA1 (h) as well as total and pS831 GluA1 (j) levels of DMSO (-) or CRT-treated hippocampal neuronal cultures. Actin served 
as a loading control. (i and k) Quantification of data shown in (h) and (j), respectively, using densitometry analysis. Data are presented as mean line density \pm SEM of CRT normalized to the control $(-)$, each dot represents one independent experiment. Statistical comparison by unpaired two-tailed ttest. The dotted line indicates the control level. ${ }^{*} \mathrm{p}<0.05,{ }^{* *} \mathrm{p}<0.01$. Scale bar $2 \mu \mathrm{m}$

\section{Fig. 3 PKD activity is required for cLTD-induced AMPAR endocytosis}

(a) Scheme displaying the cell surface biotinylation assay to detect the remaining surface GluA1 receptors after cLTD treatment. Cells were treated with DMSO or CRT followed by NMDA application. After the cLTD treatment, cultures were surface biotinylated using sulfo-NHS-SS-biotin and further processed. Representative Western Blots displaying (b) total and surface GluA1 levels, (d) PKD and pS916PKD levels, and (f) total and pS845GluA1 levels in biotinylated hippocampal neuronal cultures. Actin served as a loading control and is absent from the precipitated samples. (c, e, g) Quantification of data shown in $(b, d, f)$, using densitometry analysis. Data are presented as mean line density \pm SEM of treated samples (cLTD and CRT plus cLTD) normalized to the control; each dot represents one independent experiment. Statistical comparison was done by one-way ANOVA with Sidak's correction. The dotted lines represent the control level. (h) Interspike interval median of cells treated with $10 \mathrm{~min}$ of DMSO or CRT followed by NMDA application and a subsequent incubation period of $10 \mathrm{~min}$. Data were normalized for their pre-treatment values. The boxplots show the results of three independent experiments. Center lines show the medians; box limits indicate the $25^{\text {th }}$ and $75^{\text {th }}$ percentiles; whiskers extend 1.5 times the interquartile range from the $25^{\text {th }}$ and $75^{\text {th }}$ percentiles, outliers are represented by dots. The significance of differences was analyzed by a Mann-Whitney test (twotailed). $* * \mathrm{p}<0.01, * * * \mathrm{p}<0.001, * * * * \mathrm{p}<0.0001$.

\section{Fig. 4 Phosphorylation of Rabaptin-5 is required for cLTD-induced AMPAR endocytosis}

(a) Representative pictures of neuronal dendritic branches expressing EGFP (-), EGFP-tagged Rabaptin-5 wild-type (EGFP-Rabaptin-5 wt) or EGFP-tagged Rabaptin-5 S407A (EGFP-Rabaptin5 S/A) treated with sterile water (-) or NMDA (+). Cells were then stained for the extracellular domain of surface GluA1 and the PSD marker Shank2. Arrows point to Shank2-positive PSD areas included in the analysis. The EGFP signal is not included in the colored merged image. (b) Quantification of the images shown in (a). The boxplot shows the results of three independent experiments. Center lines show the medians; box limits indicate the $25^{\text {th }}$ and $75^{\text {th }}$ percentiles; whiskers extend 1.5 times the interquartile range from the $25^{\text {th }}$ and $75^{\text {th }}$ percentiles, outliers are represented by dots. $\mathrm{N}=769,385,835$, 323, 661 and 331 sample points. The significance of differences was analyzed by a Kruskal-Wallis test with Dunn's multiple correction. (c) Proposed model of PKD signaling in endocytic AMPAR trafficking. See text for explanation. $* p<0.05, * * p<0.01, * * * * p<0.0001$. Scale bar $1 \mu \mathrm{m}$.

\section{Supplementary figure 1 Raw MEA data}

(a) Representative phase contrast images of a MEA plate well at 13 days in vitro (DIV13) age. (b, c) Quantification of the raw interspike interval median (b) and the raw burstiness median (c) of neuronal cultures both before and after treatment with DMSO or CRT for 10 min. (d) Quantification of the raw interspike interval median of neuronal cultures both before and after treatment with DMSO or CRT for 10 minutes, followed by treatment with NMDA and a subsequent 10 min incubation. The boxplots show the results of three independent experiments. Center lines show the medians; box limits indicate the $25^{\text {th }}$ and $75^{\text {th }}$ percentiles; whiskers extend 1.5 times the interquartile range from the $25^{\text {th }}$ and $75^{\text {th }}$ percentiles, outliers are represented by dots. The significance of differences was analyzed by a 
888 Wilcoxon matched-pairs signed rank test (two-tailed). ${ }^{*} \mathrm{p}<0.05,{ }^{*} \mathrm{p}<0.01,{ }^{* * * *} \mathrm{p}<0.0001$. Scale bar $889100 \mu \mathrm{m}$.

890 Supplementary figure 2 PKD activity is required for agonist-induced AMPAR endocytosis and 891 regulates Rab5 activity through Rabaptin-5 phosphorylation

892 (a) Scheme displaying the surface biotinylation assay for receptor internalization. After surface 893 biotinylation, cells were treated with DMSO or CRT followed by AMPA and D-AP5 application. 894 Afterwards, cultures were treated with glutathione to remove remaining surface biotin. Thereby, 895 biotinylated GluA1 signal is detected only in endocytosed AMPARs, evoked by combined AMPA and 896 D-AP5 treatment. Representative Western Blots displaying (b) total and internalized GluA1 levels and 897 (d) PKD and pS916PKD levels in hippocampal neuronal cultures. Actin served as a loading control 898 and is absent from the precipitated samples. (c, e) Quantification of data shown in (b, d), using 899 densitometry analysis. Data are presented as mean line density \pm SEM of the treated samples (AMPA 900 and CRT plus AMPA) normalized to the control, each dot represents one independent experiment. 901 Statistical comparison was done by one-way ANOVA with Sidak's correction. The dotted lines 902 represent the control level. (f) Representative Western Blot displaying total and active Rab5 levels in 903 HEK293T cells transiently transfected with a plasmid encoding Rab5 in combination with either EGFP 904 (-), Rabex-5 and Rabaptin-5 wt (wt) or Rabex-5 and Rabaptin-5 S407A (S/A). Active Rab5 was 905 precipitated from the cell lysates using GST-tagged Rabaptin-5:Rab5 binding domain bound to 906 glutathione sepharose beads. (g) Quantification of data shown in (f), using densitometry analysis. Data 907 are presented as mean line density \pm SEM of the Rabaptin-5 wt and S/A samples normalized to the 908 control (EGFP), $n=5$. Statistical comparison by unpaired two-tailed $t$-test. The dotted line indicates the 909 active Rab5 level of the EGFP control. $* \mathrm{p}<0.05, * * \mathrm{p}<0.01$. 
Figure 1

A

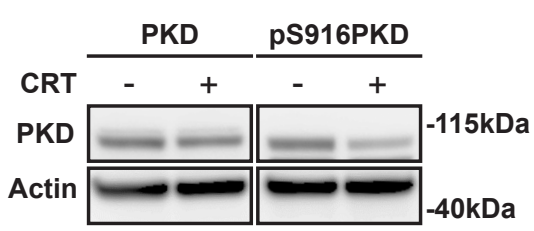

E

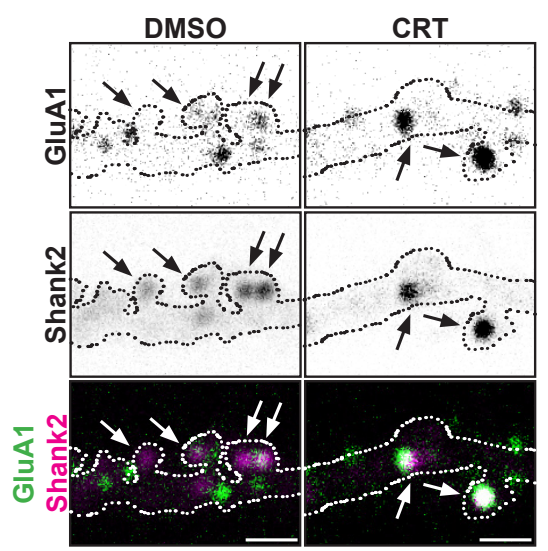

Baseline
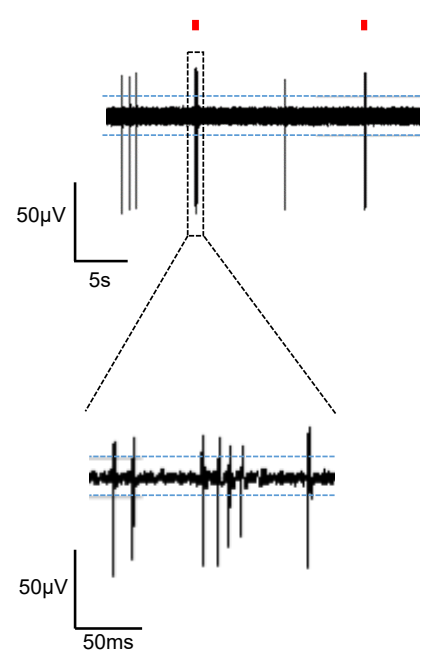

$\mathbf{F}$
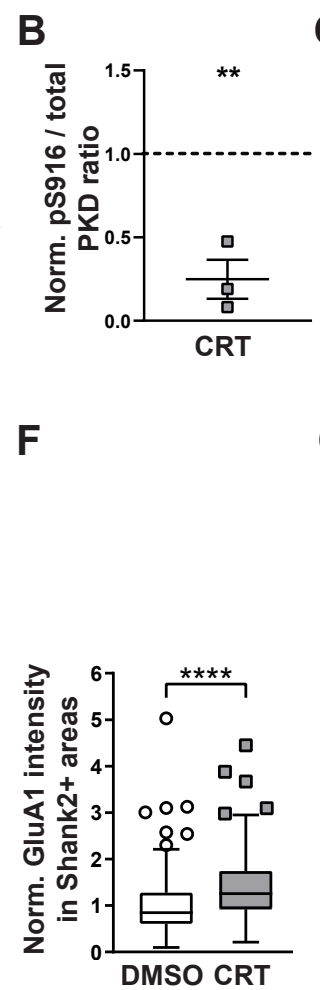

C

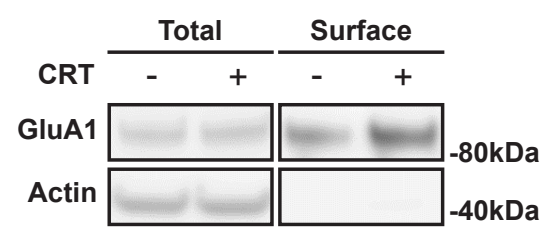

G

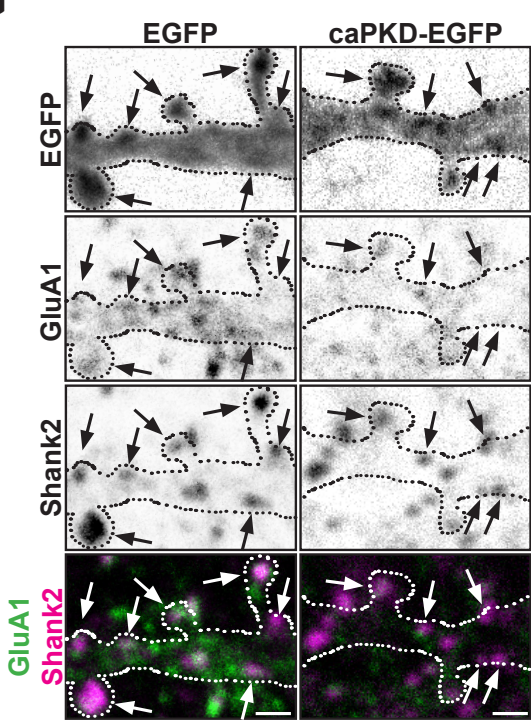

D

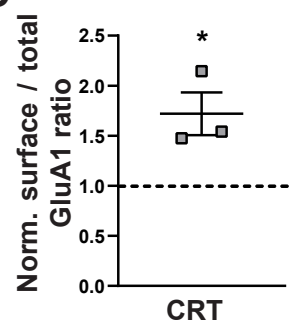

H

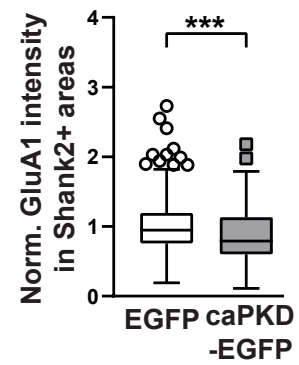

J

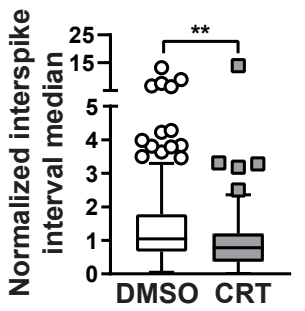

K

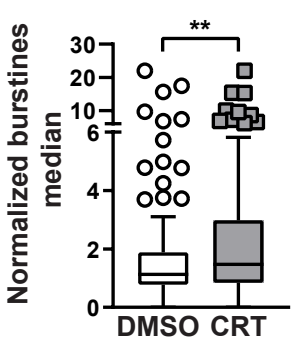




\section{Figure 2}

A
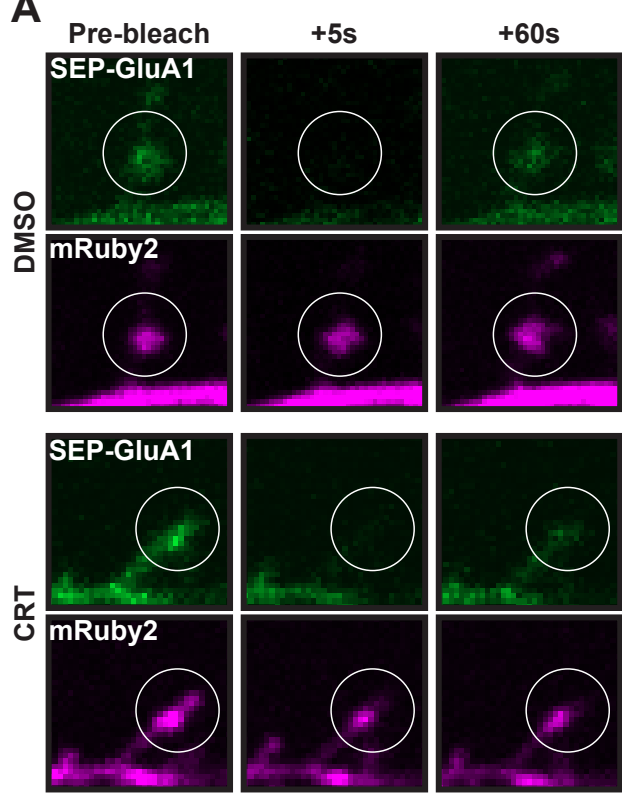

B
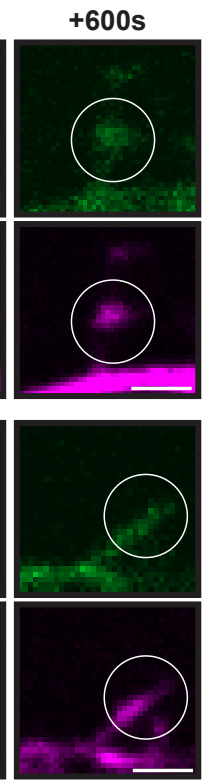

C
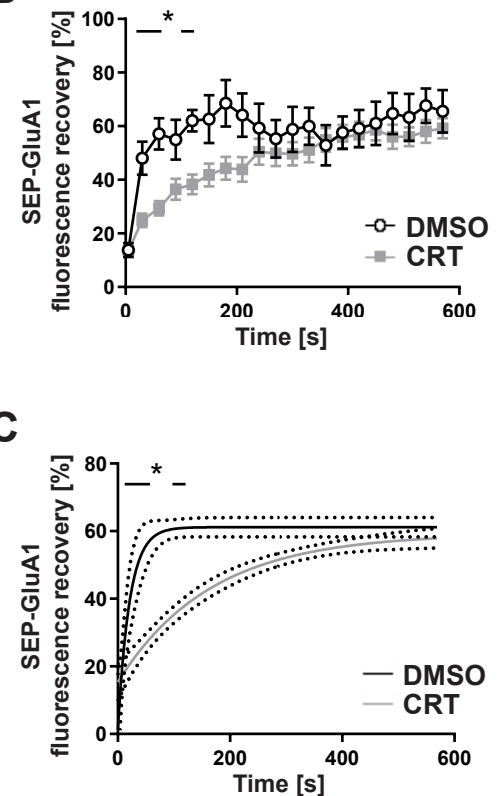

D

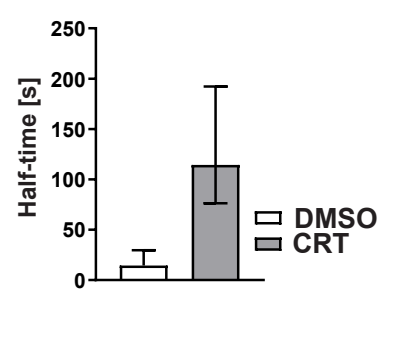

E

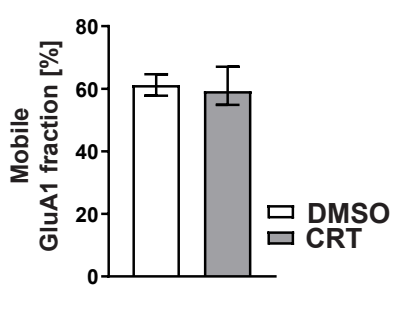

F

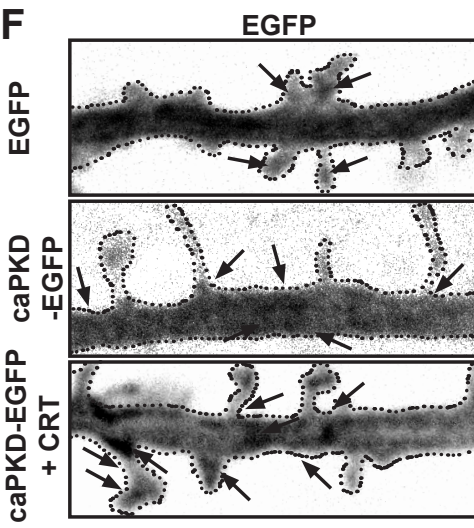

GluA1

EEA1

GIuA1 EEA1

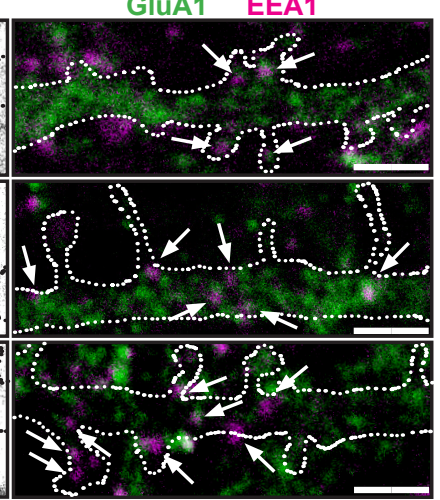

G

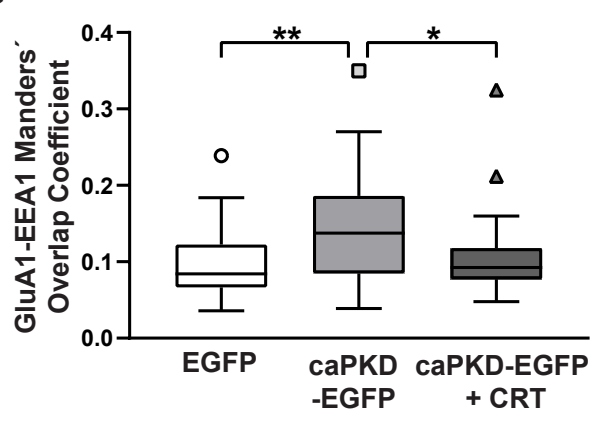

H

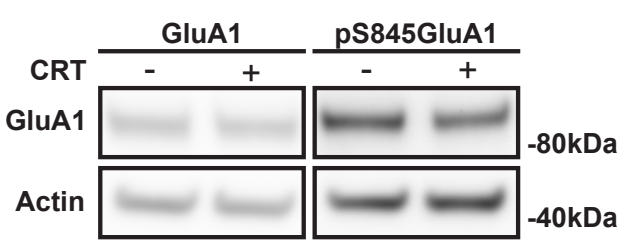

J

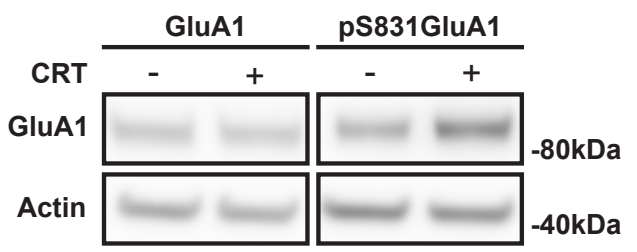

I

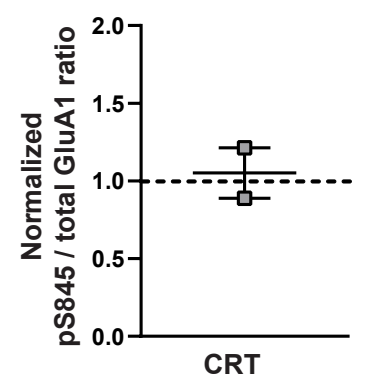

K

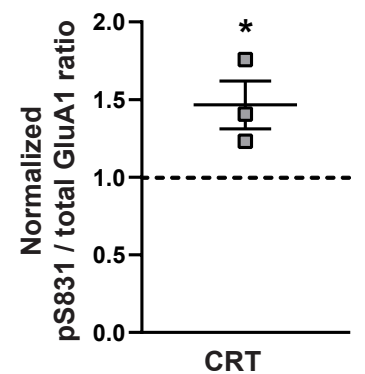




\section{Figure 3}

A
$10 \min$
5 min
$15 \min$
Sulfo-NHS-SS-Biotin
incubation
$\left(15 \mathrm{~min}, 4^{\circ} \mathrm{C}\right)$
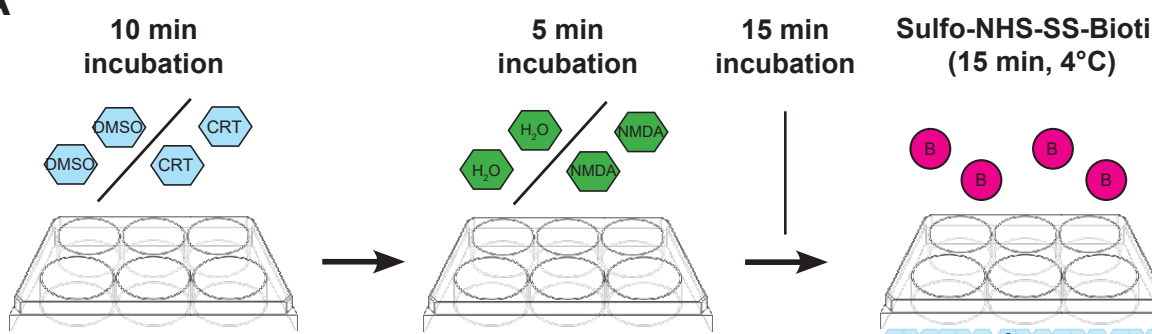

Lysis

Biotin pulldown SDS-PAGE

B

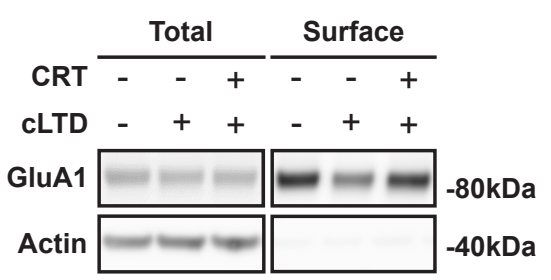

E

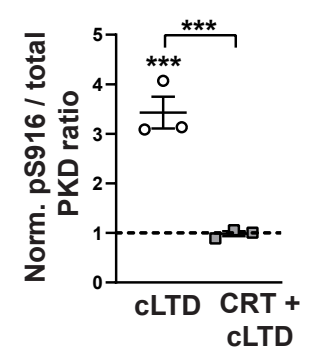

C

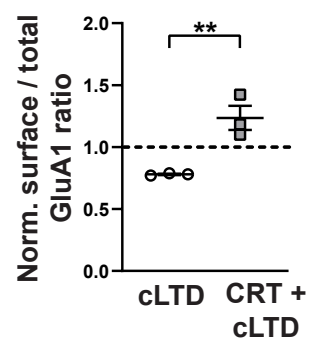

D

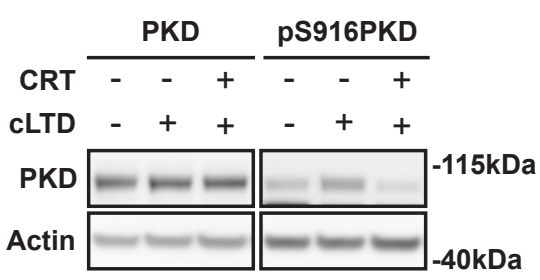

G

H

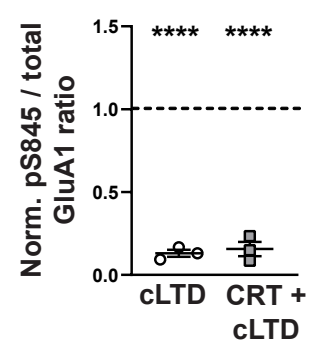

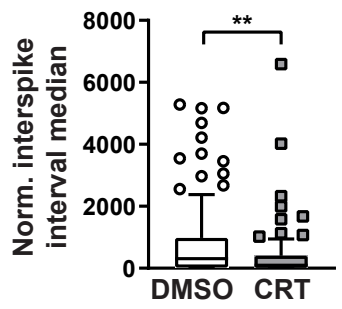




\section{Figure 4}

A

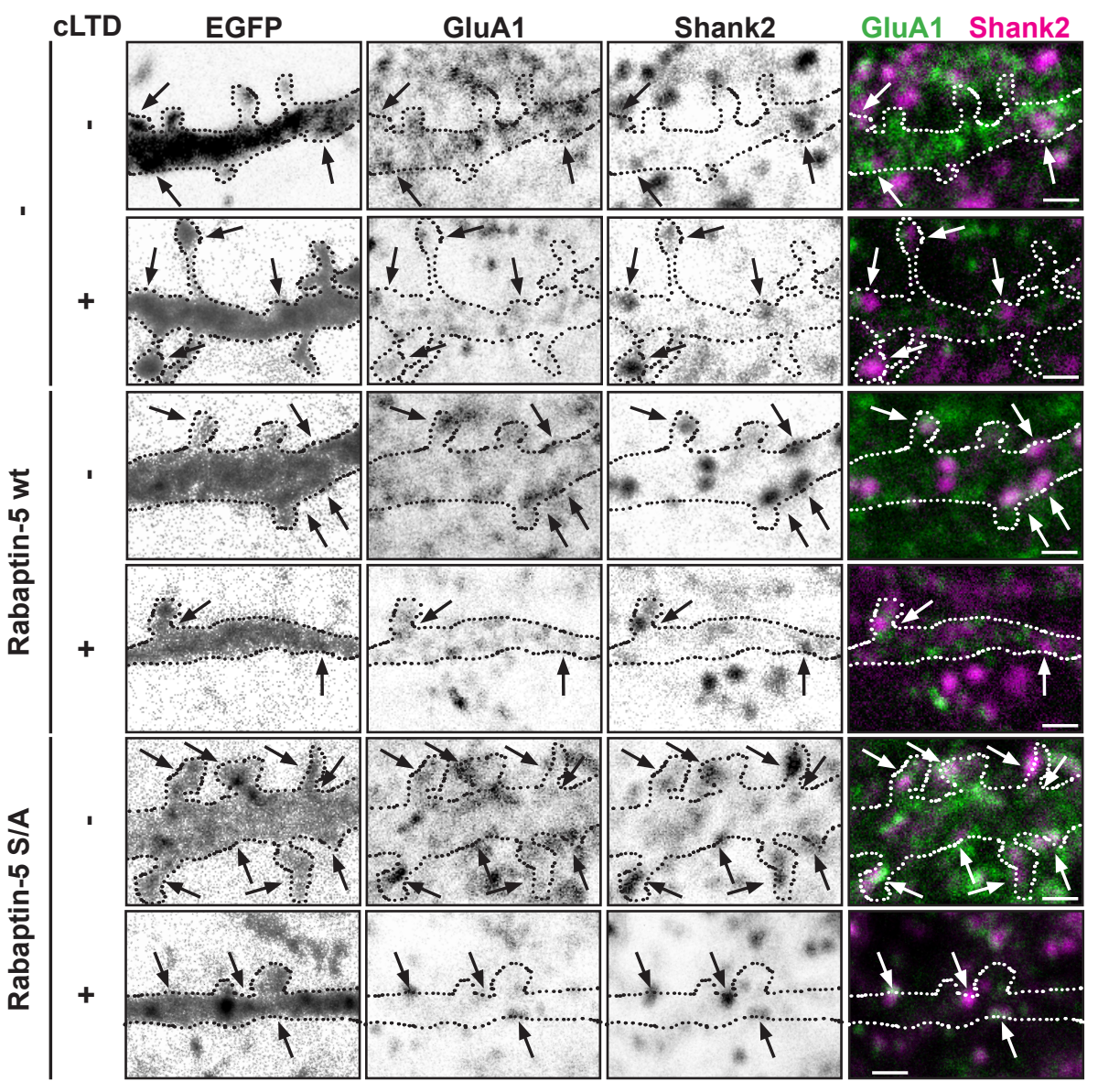

B

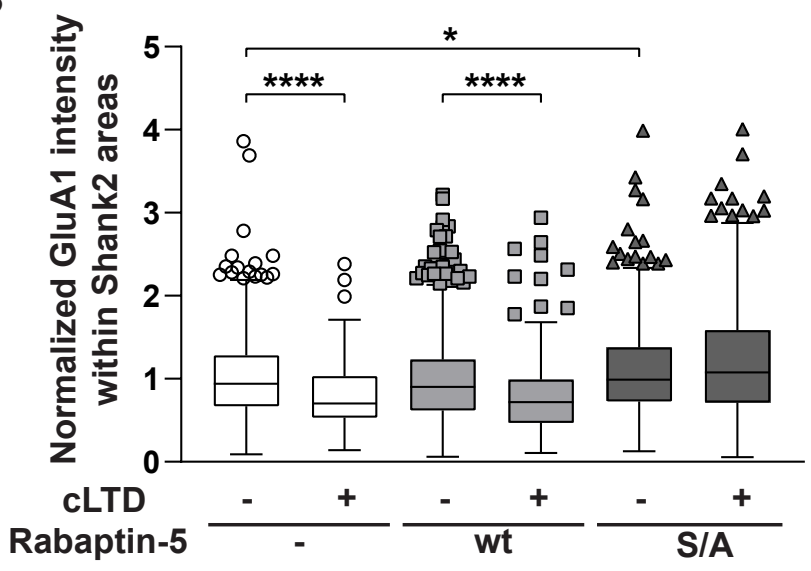

C

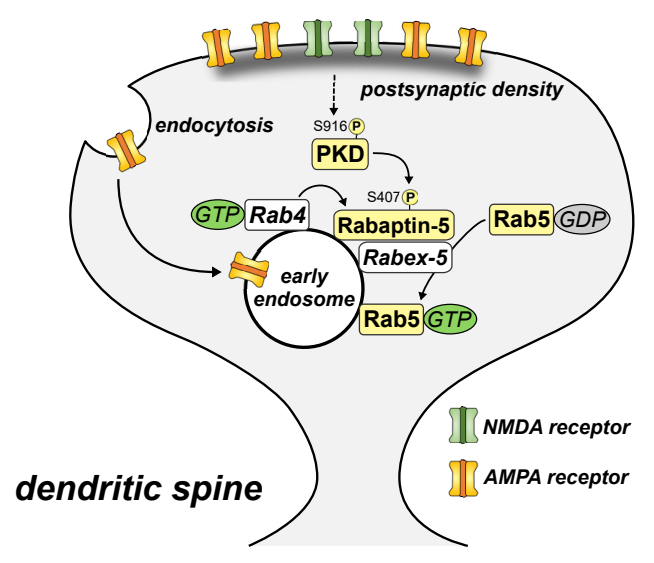




\section{Supplementary figure 1}

A
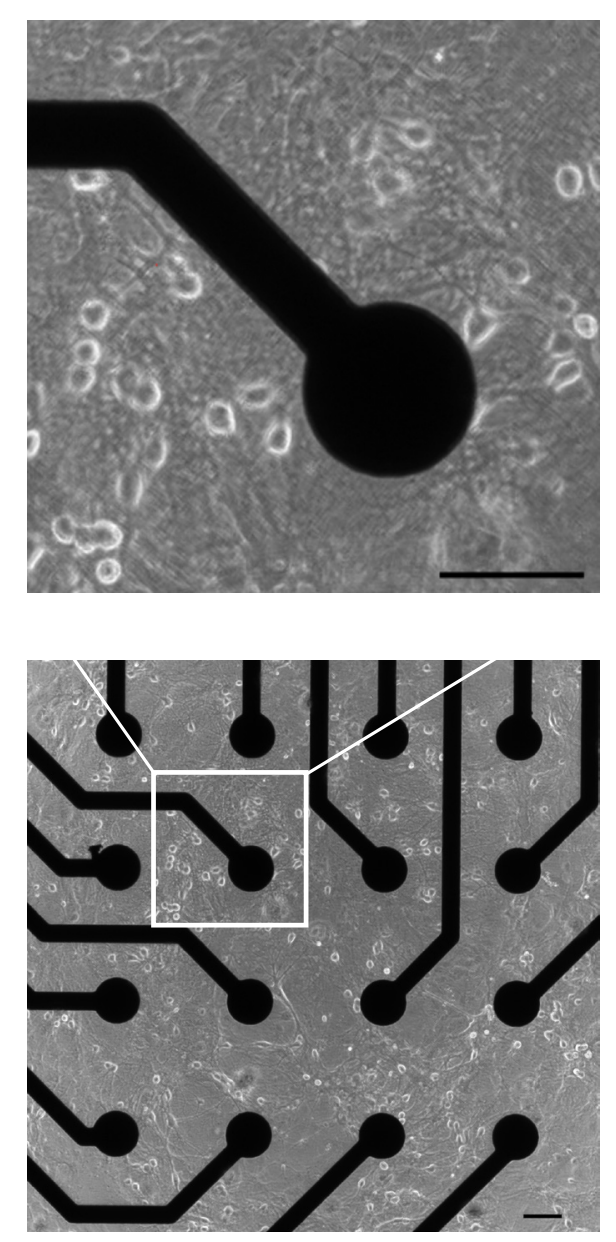

B

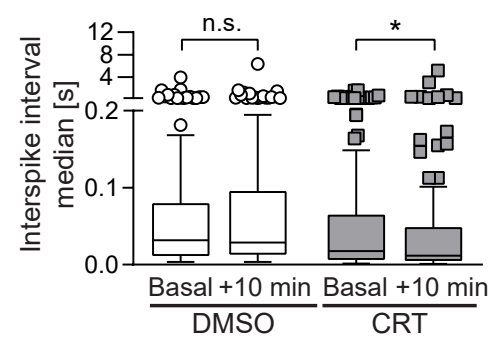

C

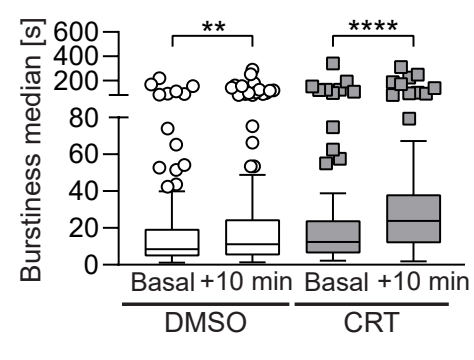

D

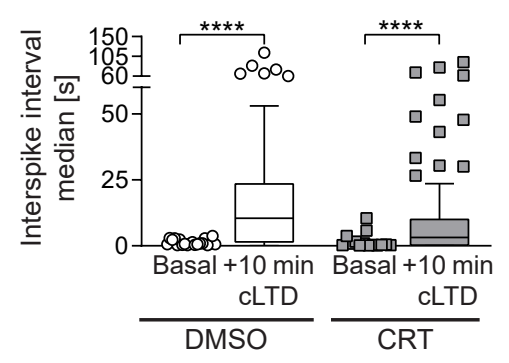




\section{Supplementary figure 2}

A

\begin{tabular}{|c|c|c|}
\hline $\begin{array}{l}\text { Sulfo-NHS-SS-Biotin } \\
\qquad\left(15 \mathrm{~min}, 4^{\circ} \mathrm{C}\right)\end{array}$ & $\begin{array}{c}10 \text { min } \\
\text { incubation }\end{array}$ & $\begin{array}{c}2 \text { min } \\
\text { incubatio }\end{array}$ \\
\hline
\end{tabular}

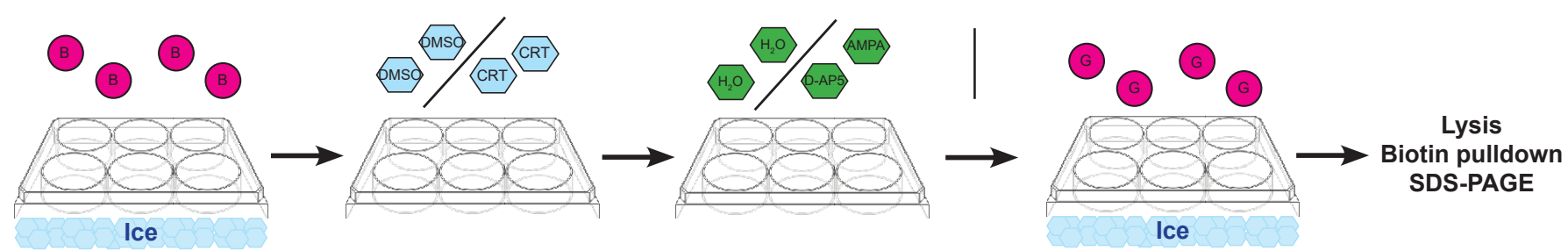

B

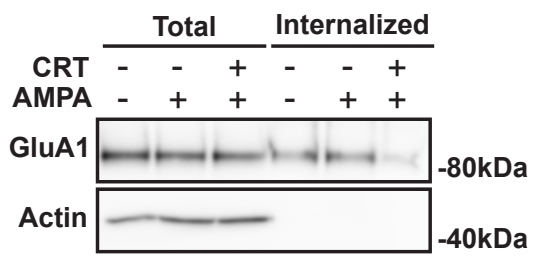

D

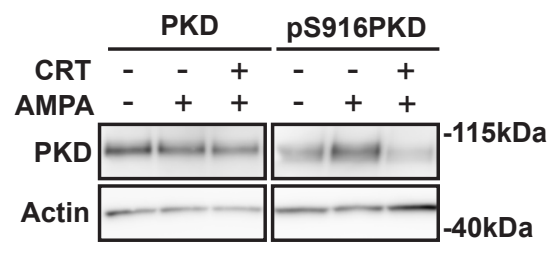

$\mathbf{F}$

\begin{tabular}{rl|ll} 
& \multicolumn{1}{c}{ Total } & \multicolumn{1}{c}{ Pulldown } \\
\cline { 2 - 3 } Rabaptin-5 & - wt S/A & - wt S/A \\
Rabaptin-5 & & & \\
Rabex-5 & & & \\
Rab5 & & & \\
\hline
\end{tabular}

C

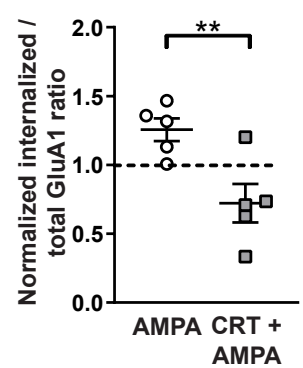

$\mathbf{E}$

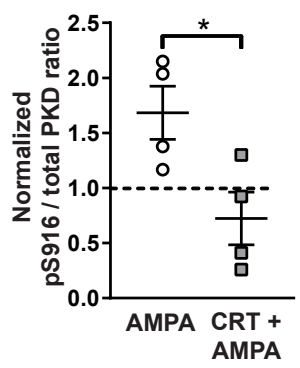

G

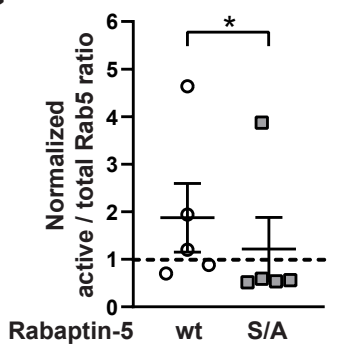

